\title{
NEW DETECTIONS OF RADIO MINIHALOS IN COOL CORES OF GALAXY CLUSTERS
}

\author{
Simona Giacintucci ${ }^{1,2}$, Maxim Markevitch $^{3,2}$, Tiziana Venturi ${ }^{4}$, Tracy E. Clarke ${ }^{5}$, \\ Rossella Cassano ${ }^{4}$, and Pasquale Mazzotta ${ }^{6,7}$ \\ ${ }^{1}$ Department of Astronomy, University of Maryland, College Park, MD 20742, USA; simona@astro.umd.edu \\ 2 Joint Space-Science Institute, University of Maryland, College Park, MD 20742-2421, USA \\ ${ }^{3}$ Astrophysics Science Division, NASA/Goddard Space Flight Center, Greenbelt, MD 20771, USA \\ ${ }^{4}$ INAF-Istituto di Radioastronomia, via Gobetti 101, I-40129 Bologna, Italy \\ ${ }^{5}$ Naval Research Laboratory, 4555 Overlook Avenue SW, Code 7213, Washington, DC 20375, USA \\ ${ }^{6}$ Dipartimento di Fisica, Universitá di Roma Tor Vergata, Via della Ricerca Scientifica 1, I-00133 Rome, Italy \\ ${ }^{7}$ Harvard-Smithsonian Center for Astrophysics, 60 Garden Street, Cambridge, MA 02138, USA \\ Received 2013 September 4; accepted 2013 November 19; published 2013 December 27
}

\begin{abstract}
Cool cores of some galaxy clusters exhibit faint radio "minihalos." Their origin is unclear, and their study has been limited by their small number. We undertook a systematic search for minihalos in a large sample of X-ray luminous clusters with high-quality radio data. In this article, we report four new minihalos (A 478, ZwCl 3146, RXJ 1532.9+3021, and A 2204) and five candidates found in the reanalyzed archival Very Large Array observations. The radio luminosities of our minihalos and candidates are in the range of $10^{23-25} \mathrm{~W} \mathrm{~Hz}^{-1}$ at $1.4 \mathrm{GHz}$, which is consistent with these types of radio sources. Their sizes (40-160 kpc in radius) are somewhat smaller than those of previously known minihalos. We combine our new detections with previously known minihalos, obtaining a total sample of 21 objects, and briefly compare the cluster radio properties to the average X-ray temperature and the total masses estimated from Planck. We find that nearly all clusters hosting minihalos are hot and massive. Beyond that, there is no clear correlation between the minihalo radio power and cluster temperature or mass (in contrast with the giant radio halos found in cluster mergers, whose radio luminosity correlates with the cluster mass). Chandra $\mathrm{X}$-ray images indicate gas sloshing in the cool cores of most of our clusters, with minihalos contained within the sloshing regions in many of them. This supports the hypothesis that radio-emitting electrons are reaccelerated by sloshing. Advection of relativistic electrons by the sloshing gas may also play a role in the formation of the less extended minihalos.
\end{abstract}

Key words: galaxies: clusters: general - galaxies: clusters: individual (MACSJ0159.8-0849, MACSJ0329.6-0211, A478, ZwCl3146, A1795) - galaxies: clusters: intracluster medium - radio continuum: general - X-rays: galaxies: clusters

Online-only material: color figures

\section{INTRODUCTION}

A number of relaxed, cool-core clusters of galaxies are known to host centrally located, diffuse synchrotron radio emission in their cores that typically fills the cooling region $(r \sim 50-300 \mathrm{kpc})$. These extended radio sources-called minihalos-have very low surface brightness and possess steep radio spectra, $\alpha>1$ (for $S_{v} \propto v^{-\alpha}$ ). Their emission usually encompasses the radio source associated with the cluster dominant galaxy, but it extends to much greater radii. Only about 10 clusters have confirmed minihalos (Feretti et al. 2012 and references therein). These include the prototype minihalo in the Perseus cluster (Burns et al. 1992; Pedlar et al. 1990; Sijbring 1993), whose emission occupies a large volume of the cool core, and it is much larger than the inner $r \sim 30 \mathrm{kpc}$ region occupied by the well-known X-ray cavities filled by the lobes of 3C 84 (e.g., Fabian et al. 2011).

The origin of radio minihalos is unclear. Even though minihalos have active galaxies in their centers, the time needed by the electrons to diffuse from the galaxy across the cooling region $\left(\sim 10^{9} \mathrm{yr}\right)$ is much longer than the radiative cooling timescale $\left(\sim 10^{7}-10^{8}\right.$ yr) for $\gamma \sim 10^{4}$ electrons that emit at the observed radio frequencies in the strong magnetic fields of the cool cores (e.g., Taylor et al. 2002). Thus, in situ production or reacceleration of relativistic electrons is required to explain minihalos. One possibility is inelastic collisions between relativistic cosmic ray protons and thermal protons, which can provide a continuous injection of relativistic electrons ("secondary" or "hadronic" models; Pfrommer \& Enßlin 2004; Fujita et al. 2007; Keshet \& Loeb 2010; Keshet 2010; Fujita \& Ohira 2013). Alternatively, reacceleration models (Gitti et al. 2002, 2004) posit that the radio synchrotron emission arises from preexisting, cooled relativistic electrons (for instance, injected by past activity of the central radio galaxy and/or hadronic collisions) that are reaccelerated to ultrarelativistic energies by turbulence in the intracluster medium (ICM).

A key question for the latter scenario is the origin of the turbulence responsible for accelerating the electrons. An interesting possibility is that it is induced by sloshing gas motions, detected using high-resolution X-ray observations in the cool cores of most relaxed clusters. The observational signature of these motions is the spiral- or arc-shaped cold fronts (e.g., Markevitch et al. 2001; Mazzotta et al. 2001, 2003; Markevitch \& Vikhlinin 2007; Owers et al. 2009; Ghizzardi et al. 2010; Laganá et al. 2010). These are believed to be contact discontinuities at the boundaries of the dense cool core that is sloshing in the deep potential well of the cluster in response to, e.g., a gravitational perturbation caused by the infall of a small subcluster (Ascasibar \& Markevitch 2006). Numerical simulations have shown that sloshing can generate significant turbulence in the core (Fujita et al. 2004; Vazza et al. 2012; ZuHone et al. 2013). 
The existence of a possible connection between gas sloshing and radio minihalos was first hinted at by the discovery of a spatial correlation of the minihalo with X-ray cold fronts in two clusters (Mazzotta \& Giacintucci 2008), where the diffuse radio emission appears confined to the region bounded by the fronts. A similar correlation is also observed in the Perseus cluster (M. Markevitch et al., in preparation). ZuHone et al. (2013) used simulations to test the possibility of accelerating relativistic electrons via sloshing-driven turbulence. Though theoretical uncertainties are large, they found that turbulence can be strong enough to reaccelerate low- $\gamma$ electrons to higher energies $\left(\gamma \sim 10^{4}\right)$ and produce diffuse radio emission with morphology, radio power, and spectral index consistent with actual minihalos.

However, despite theoretical effort, our understanding of the origin and physical properties of minihalos and their relation to the cluster core dynamics is still limited by the small number of known objects. We undertook a search for new minihalos in a large sample of galaxy clusters that combines radio observations from the Very Large Array (VLA) archive and the Giant Metrewave Radio Telescope (GMRT; proprietary and archival), along with X-ray data from the Chandra archive. In this article we present new minihalos (and several candidates requiring better data for confirmation) found during reanalysis of the VLA observations. Statistical analysis of the cluster sample and detailed radio/X-ray study of specific interesting cases will be presented in forthcoming articles.

We adopt $\Lambda C D M$ cosmology with $H_{0}=70 \mathrm{~km} \mathrm{~s}^{-1} \mathrm{Mpc}^{-1}$, $\Omega_{m}=0.3$, and $\Omega_{\Lambda}=0.7$.

\section{RADIO OBSERVATIONS AND DATA REDUCTION}

In this section we briefly give the relevant information on our cluster sample in which we searched for minihalos. More details on the sample selection will be presented in a future article about correlations of various cluster properties. We selected all those clusters from the Chandra $\mathrm{ACCEPT}^{8}$ sample (Cavagnolo et al. 2009), which contain deep, pointed radio observations suitable to look for a central minihalo or place an interesting upper limit on it, obtaining a sample of $\sim 100$ clusters. The radio information was obtained from the VLA archive and/or proprietary GMRT data, mostly from the GMRT Radio Halo Survey (GRHS; Venturi et al. 2007, 2008) and its recent extension (eGRHS; Kale et al. 2013). We considered only clusters at $z \leqslant 0.5$ and $\mathrm{DEC}_{\mathrm{J} 2000}>-30^{\circ}$, to ensure a good sampling of the $u-v$ plane of the radio observations, which was necessary to properly image extended and complex radio sources such as minihalos. A minihalo has previously been reported for 12 clusters of our sample. In this article, we report detections of new minihalos or candidates for nine more clusters (listed in Table 1), based on reanalysis of the VLA archival observations. The VLA observations are summarized in Table 2.

The data were calibrated and reduced using the NRAO AIPS. Images were produced using the standard Fourier transform deconvolution method. Self-calibration was applied to reduce the effects of residual phase errors in the data and to improve the quality of the final images. Correction for the primary beam attenuation was applied to the images using the task PBCOR in AIPS. All flux densities are in the Perley \& Butler (2013) scale, and residual amplitude errors are within $5 \%$ at all frequencies.

\footnotetext{
8 Archive of Chandra Cluster Entropy Profile Tables.
}

Table 1

List of Clusters

\begin{tabular}{lcccc}
\hline \hline Cluster name & $\begin{array}{c}\text { R.A.J2000 } \\
(\mathrm{h}, \mathrm{m}, \mathrm{s})\end{array}$ & $\begin{array}{c}\text { Decl.J2000 } \\
\left({ }^{\circ},{ }^{\prime},{ }^{\prime \prime}\right)\end{array}$ & $z$ & $\begin{array}{c}\text { scale } \\
\left(\mathrm{kpc} /{ }^{\prime \prime}\right)\end{array}$ \\
\hline MACS J0159.8-0849 & 015948.0 & -084900 & 0.405 & 5.413 \\
MACS J0329.6-0211 & 032940.8 & -021154 & 0.450 & 5.759 \\
A 478 & 041320.7 & +102835 & 0.088 & 1.646 \\
ZwCl 3146 & 102339.6 & +041110 & 0.290 & 4.350 \\
A 1795 & 134900.5 & +263507 & 0.062 & 1.195 \\
RX J1532.9+3021 & 153254.4 & +302111 & $0.362^{\mathrm{a}}$ & 5.048 \\
A 2204 & 163245.7 & +053443 & 0.152 & 2.643 \\
ZwCl 1742.1+3306 & 174413.5 & +325855 & 0.076 & 1.441 \\
MACS J1931.8-2635 & 193148.0 & -263500 & 0.352 & 4.958 \\
\hline
\end{tabular}

Notes. Column 1: cluster name. Columns 2-4: cluster coordinates and redshift from the NASA/IPAC Extragalactic Database (NED). Column 5: angular to linear scale conversion.

a Spectroscopic redshift of the BCG from the Sloan Digital Sky Survey Data Release 9 (SDSS DR9). A redshift of $z=0.345$ was initially reported in the ROSAT Brightest Cluster Sample (Ebeling et al. 1998), but later optical followup established that the cluster is at $z=0.3615$ (Crawford et al. 1999).

\section{RADIO AND X-RAY DATA}

The VLA setup for our clusters is presented in Table 2. For almost all clusters, it is possible to image the central radio emission at different angular resolutions. The higher-resolution images are used to identify the radio source associated with the brightest cluster galaxy (BCG) and other possible radio galaxies in (or projected onto) the cluster core region. Low-resolution images are then used to map the diffuse radio emission. The radio images are compared to the X-ray Chandra images to search for possible indications of a spatial correlation of the diffuse radio emission with the ICM substructures (e.g., cold fronts, surface brightness edges, and spiral-like features). A detailed study of any radio/X-ray surface brightness correlation found here will be presented in future articles. Table 3 lists the Chandra observations used to produce the X-ray images. As we are only interested in a qualitative analysis of the core structure, for most of our clusters we extracted images directly from the standard event files produced and archived by the Chandra $\mathrm{X}$-ray Center, without additional data filtering, background subtraction, or exposure map correction. For RX J1532.9+3021, we use an exposure-map corrected image that combines three available observations for a total clean exposure of $105 \mathrm{ks}$ (courtesy of J. Hlavacek-Larrondo; for details see HlavacekLarrondo et al. 2013).

We summarize the position and flux densities of the radio galaxies in each cluster image, their total spectral index, and their optical identification in Table 4. In Table 5, we summarize the radio properties of the new minihalos and candidates presented in this article. For all clusters, we determined the position and flux density of the unresolved radio galaxies by fitting the sources with a Gaussian model (task JMFIT in AIPS).

For extended radio galaxies, we measured the total flux density by integration within the $+3 \sigma$ surface brightness contour using the task TVSTAT on the final images. For the minihalos, we measured the total flux in circular regions of a radius that progressively increased from the radius encompassing the $+3 \sigma$ isocontour until the integrated flux density reached saturation. For most minihalos, more than $95 \%$ of the flux is contained already within the $+3 \sigma$-isocontour radius. 
Table 2

Details of the VLA Observations

\begin{tabular}{|c|c|c|c|c|c|c|c|c|}
\hline Cluster Name & Project & Array & $\begin{array}{c}\text { Frequency } \\
(\mathrm{GHz})\end{array}$ & $\begin{array}{c}\text { Bandwidth } \\
(\mathrm{MHz})\end{array}$ & Date & $\begin{array}{l}\text { Time } \\
(\min )\end{array}$ & $\begin{array}{c}\text { FWHM, PA } \\
\left({ }^{\prime \prime} \times^{\prime \prime},{ }^{\circ}\right)\end{array}$ & $\begin{array}{c}\mathrm{rms} \\
\left(\mu \mathrm{Jy} \mathrm{b}^{-1}\right)\end{array}$ \\
\hline \multirow[t]{2}{*}{ MACS J0159.8-0849 } & $\mathrm{AE} 147$ & $\mathrm{~B}$ & 1.4 & 50 & $2002 \mathrm{Jul} 15$ & 48 & $5.7 \times 4.2,-17$ & 15 \\
\hline & AE117 & $\mathrm{A}$ & 8.5 & 50 & 1998 Apr 12 & 19 & $0.3 \times 0.2,-28$ & 18 \\
\hline MACS J0329.6-0211 & AE142 & B & 1.4 & 50 & 2001 May 05 & 48 & $4.7 \times 4.5,0$ & 40 \\
\hline \multirow[t]{3}{*}{ A 478} & AB 1150 & A & 1.4 & 25 & 2004 Nov 24 & 320 & $1.3 \times 1.3,-12$ & 25 \\
\hline & AM938 & $\mathrm{C}$ & 1.4 & 50 & 2009 Jul 18 & 190 & $14.2 \times 11.8,-49$ & 40 \\
\hline & AK685 & $\mathrm{C}$ & 4.9 & 50 & 2008 May 19 & 93 & $4.1 \times 3.8,-29$ & 15 \\
\hline \multirow[t]{2}{*}{ ZwCl 3146} & AB 1190 & $\mathrm{C}$ & 4.9 & 50 & 2006 Dec 18 & 272 & $4.3 \times 3.9,40$ & 10 \\
\hline & AB1190 & $\mathrm{C}$ & 8.5 & 50 & 2006 Dec 18 & 102 & $2.5 \times 2.4,-34$ & 15 \\
\hline \multirow[t]{2}{*}{ A 1795} & AO84 & A & 1.4 & 3 & 1989 Jan 18 & 176 & $1.3 \times 1.2,69$ & 40 \\
\hline & AJ215 & $\mathrm{C}$ & 1.4 & 6 & 1992 Feb 25 & 130 & $14.1 \times 13.4,40$ & 150 \\
\hline \multirow[t]{7}{*}{ RX J1532.9+3021 } & AT0318 & A & 1.4 & 50 & 2006 Mar 15 & 72 & $1.4 \times 1.1,-29$ & 12 \\
\hline & AT0318 & B & 1.4 & 50 & 2006 Sep 11 & 50 & $4.2 \times 3.7,-11$ & 15 \\
\hline & AT0318 & B & 0.3 & 6 & 2006 Mar 15 & 118 & $5.2 \times 4.8,-27$ & 500 \\
\hline & AE110 & $\mathrm{C}$ & 4.9 & 50 & 1997 Jun 26 & 6 & $4.8 \times 3.9,-75$ & 20 \\
\hline & AE117 & A & 8.5 & 50 & 1998 Apr 12 & 11 & $0.2 \times 0.2,42$ & 25 \\
\hline & AK633 & $\mathrm{D}$ & 8.5 & 50 & 2005 Apr 7 & 5 & $8.1 \times 7.4,-73$ & 30 \\
\hline & AK633 & $\mathrm{D}$ & 22 & 50 & 2007 Apr 5 & 4 & $2.9 \times 2.6,-75$ & 140 \\
\hline \multirow[t]{2}{*}{ A 2204} & AT0211 & A & 1.4 & 25 & 1998 Apr 23 & 123 & $1.3 \times 1.3,1$ & 30 \\
\hline & S8398 & B & 1.4 & 50 & 2007 Nov 25 & 102 & $4.7 \times 4.2,38$ & 30 \\
\hline ZwCl 1742.1+3306 & AE130 & DnA & 1.4 & 6 & 1999 Jun 10 & 145 & $1.5 \times 1.5,47$ & 65 \\
\hline \multirow[t]{3}{*}{ MACS J1931.8-2634 } & AT0318 & A & 1.4 & 50 & 2006 Apr 14 & 82 & $2.5 \times 1.2,3$ & 30 \\
\hline & AT0318 & B & 1.4 & 50 & 2006 Sep 09 & 49 & $8.4 \times 3.8,0$ & 40 \\
\hline & AT0318 & A & 0.3 & 6.3 & 2006 Apr 14 & 120 & $11.0 \times 4.5,1$ & 1700 \\
\hline
\end{tabular}

Notes. Column 1: cluster name. Columns 2 and 3: VLA array configuration and project. Columns 4-6: observing frequency, total bandwidth, and date. Column 7: total time on source. Column 8: full width at half-maximun (FWHM) and position angle (PA) of the array. Column 9: image rms level (1 $\sigma$ ).

Table 3

List of the Chandra Observations

\begin{tabular}{lccc}
\hline \hline Cluster Name & Detector & Observation ID & $\begin{array}{c}\text { Exposure }^{\mathrm{a}} \\
(\mathrm{ks})\end{array}$ \\
\hline MACS J0159.8-0849 & ACIS-I & $3265,6106,9376$ & 70 \\
MACS J0329.6-0211 & ACIS-I & $3257,3582,6108$ & 70 \\
A 478 & ACIS-S & 1669 & 40 \\
ZwCl 3146 & ACIS-I & 909,9371 & 90 \\
A 1795 & ACIS-S & 493,494 & 40 \\
RX J1532.9+3021 & ACIS-S, I, S & $1649,1665,14009$ & $105^{\mathrm{b}}$ \\
A 2204 & ACIS-S, I, I & $499,6104,7940$ & 100 \\
ZwCl 1742.1+3306 & ACIS-S & 11708 & 45 \\
MACS J1931.8-2635 & ACIS-I & 9382 & 100 \\
\hline
\end{tabular}

Notes.

a Total exposure time without filtering.

b Total clean exposure from Hlavacek-Larrondo et al. (2013).

Following Cassano et al. (2013), we estimated the error on the minihalo flux density $S_{\mathrm{MH}}$ as

$$
\sigma_{S_{\mathrm{MH}}}=\sqrt{\left(\sigma_{\mathrm{cal}} S_{\mathrm{MH}}\right)^{2}+\left(\mathrm{rms} \sqrt{N_{\text {beam }}}\right)^{2}+\sigma_{\mathrm{sub}}^{2}},
$$

which takes into account the uncertainty on the flux density scale $\left(\sigma_{\text {cal }} \sim 5 \%\right)$, the image rms level weighted by the number of beams in the minihalo region $\left(N_{\text {beam }}\right)$, and the uncertainty $\sigma_{\text {sub }}$ in the subtraction of the flux density of the embedded discrete radio sources from the total flux density measured in the image. This latter is estimated as

$$
\sigma_{\mathrm{sub}}^{2}=\sum_{i=1}^{N}\left(I_{\mathrm{MH}, i} \times A_{s, i}\right)^{2},
$$

where $I_{H, i}$ is the average surface brightness of the minihalo in the region of the $i$-th radio source, occupying an area $A_{s, i}$.
Because of the irregular and nonspherical morphology of some minihalos, we estimated the average radius of the diffuse emission as

$$
R_{\mathrm{MH}}=\sqrt{R_{\max } \times R_{\min }},
$$

where $R_{\max }$ and $R_{\min }$ are the maximum and minimum radius as derived from the $+3 \sigma$ isocontour in the images (Cassano et al. 2007). However, the sizes determined in this way may be affected by the different signal-to-noise ratios of the images and should therefore be considered as a lower limit for the real sizes. A more appropriate definition of the size, independent on the sensitivity of the observations, should be used for quantitative studies of the minihalo properties (e.g., Murgia et al. 2009). We will address this in a future article by analyzing the surface brightness radial profiles of all minihalos, including the new detections presented here.

In Table 5, we also provide the list of the clusters hosting known minihalos. For consistency with the present work, we have reanalyzed the existing VLA archival observations of all these systems (except for Perseus and A 2626, for which we use the literature information as reported in the table) and rederived the minihalo flux densities and sizes following the procedure described above (S. Giacintucci et al., in preparation). We note that our list does not include the clusters A 2142 and MRC 0116+111 in which minihalos have been reported in the literature. Recent Green Bank Telescope (GBT) observations at $1.4 \mathrm{GHz}$ (Farnsworth et al. 2013; Rossetti et al. 2013) and GMRT observations at lower frequencies (T. Venturi et al., in preparation) reveal that the central diffuse source A 2142 is far more extended than the $\sim 200 \mathrm{kpc}$ minihalo imaged by Giovannini \& Feretti (2000), covering a scale of the order of the Mpc, and thus falls into the category of "giant" radio halos (e.g., Feretti et al. 2012). No X-ray information is available for MRC $0116+111$, thus the classification of the source as a 
Table 4

Properties of the Discrete Radio Sources

\begin{tabular}{|c|c|c|c|c|c|c|c|}
\hline Cluster Name & Source & $\begin{array}{c}\text { R.A.J2000 } \\
(\mathrm{h}, \mathrm{m}, \mathrm{s})\end{array}$ & $\begin{array}{c}\text { Decl.J2000 } \\
\left({ }^{\circ},{ }^{\prime},{ }^{\prime \prime}\right)\end{array}$ & $\begin{array}{c}v \\
(\mathrm{GHz})\end{array}$ & $\begin{array}{c}S_{v} \\
(\mathrm{mJy})\end{array}$ & $\alpha$ & Notes \\
\hline \multirow[t]{3}{*}{ MACS J0159.8-0849 } & $\mathrm{S} 1$ & 015949.3 & -084959 & 1.4 & $35.0 \pm 1.8$ & $-0.55 \pm 0.04$ & $\mathrm{BCG}$ \\
\hline & & & & 8.5 & $94.8 \pm 4.8$ & & \\
\hline & $\mathrm{S} 2$ & 015950.9 & -084950 & 1.4 & $0.43 \pm 0.03$ & $\ldots$ & Possible member galaxy ${ }^{a}$ \\
\hline MACS J0329.6-0211 & $\mathrm{S} 1$ & 032941.6 & -021147 & 1.4 & $3.8 \pm 0.2$ & $\ldots$ & $\mathrm{BCG}$ \\
\hline \multirow[t]{7}{*}{ A 478} & $\mathrm{~S} 1$ & 041325.3 & +102755 & 1.4 & $31.0 \pm 1.6$ & $0.92 \pm 0.06$ & $\mathrm{BCG}$ \\
\hline & & & & 4.9 & $9.6 \pm 0.5$ & & \\
\hline & $\mathrm{S} 2$ & 041331.8 & +102839 & 1.4 & $2.5 \pm 0.1$ & $1.03 \pm 0.06$ & Member galaxy ${ }^{b}$ \\
\hline & & & & 4.9 & $0.68 \pm 0.04$ & & \\
\hline & S3 & 041335.2 & +102921 & 1.4 & $1.10 \pm 0.06$ & $-0.08 \pm 0.06$ & no $z$ \\
\hline & S4 & 041338.3 & +102809 & 1.4 & $47.4 \pm 2.4$ & $0.93 \pm 0.05$ & Member galaxy ${ }^{\mathrm{c}}$ \\
\hline & & & & 4.9 & $14.6 \pm 0.7$ & & \\
\hline \multirow[t]{6}{*}{ ZwCl 3146} & $\mathrm{~S} 1$ & 102339.6 & +041111 & 1.4 & $\sim 3.3^{\mathrm{d}}$ & $0.67 \pm 0.10^{\mathrm{e}}$ & $\mathrm{BCG}$ \\
\hline & & & & 4.9 & $1.42 \pm 0.07$ & & \\
\hline & & & & 8.5 & $0.98 \pm 0.03$ & & \\
\hline & $\mathrm{S} 2$ & 102338.7 & +041105 & 1.4 & $0.2^{\mathrm{d}}$ & $-0.32 \pm 0.15^{\mathrm{e}}$ & Background galaxy ${ }^{\mathrm{f}}$ \\
\hline & & & & 4.9 & $0.31 \pm 0.02$ & & \\
\hline & & & & 8.5 & $0.37 \pm 0.02$ & & \\
\hline \multirow[t]{2}{*}{ A 1795} & $\mathrm{~S} 1$ & 134852.5 & +263534 & 1.4 & $917 \pm 46^{\mathrm{g}}$ & & $\mathrm{BCG}$ \\
\hline & $\mathrm{S} 2$ & 134845.5 & +263524 & 1.4 & $5.1 \pm 0.3$ & & Background galaxy ${ }^{\mathrm{h}}$ \\
\hline \multirow[t]{6}{*}{ RX J1532.9+3021 } & $\mathrm{S} 1$ & 153253.8 & +302059 & 0.3 & $26.3 \pm 1.4$ & $0.43 \pm 0.02$ & $\mathrm{BCG}$ \\
\hline & & & & $0.6^{\mathrm{i}}$ & $20.3 \pm 1.0$ & & \\
\hline & & & & 1.4 & $15.7 \pm 0.8$ & & \\
\hline & & & & 4.9 & $8.8 \pm 0.4$ & & \\
\hline & & & & 8.5 & $7.0 \pm 0.4$ & & \\
\hline & & & & 22 & $4.2 \pm 0.2$ & & \\
\hline \multirow[t]{2}{*}{ A 2204} & $\mathrm{~S} 1$ & 163247.0 & +053435 & 1.4 & $58.9 \pm 2.9$ & $\ldots$ & $\mathrm{BCG}$ \\
\hline & $\mathrm{S} 2$ & 163247.0 & +053441 & 1.4 & $1.6 \pm 0.1$ & $\ldots$ & No $z$ \\
\hline \multirow[t]{2}{*}{$\mathrm{ZwCl} 1742.1+3306$} & $\mathrm{~S} 1$ & 174414.5 & 325929 & 1.4 & $68.8 \pm 3.4$ & $\ldots$ & BCG \\
\hline & $\mathrm{S} 2$ & 174417.1 & 325914 & 1.4 & $0.94 \pm 0.05$ & $\ldots$ & No $z$ \\
\hline \multirow[t]{2}{*}{ MACS J1931.8-2634 } & $\mathrm{S} 1$ & & & 1.4 & $11.6 \pm 0.05$ & & BCG \\
\hline & $\mathrm{S} 2$ & & & 1.4 & $2.5 \pm 0.1$ & & No opt. id. \\
\hline
\end{tabular}

Notes. Column 1: cluster name. Column 2: radio source. Columns 3 and 4: radio coordinates. Column 5: frequency. Column 6: flux density. Column 7: spectral index. Column 8: notes on the optical identification.

${ }^{\mathrm{a}} z_{\text {phot }}=0.44 \pm 0.04$ from SDSS DR9.

b $z=0.0894 \pm 0.0003$ (Zabludoff et al. 1990).

c $z=0.0934 \pm 0.0002$ (Zabludoff et al. 1990).

${ }^{\mathrm{d}}$ Expected value based on $\alpha=0.67$ between $4.9 \mathrm{GHz}$ and $8.5 \mathrm{GHz}$.

e Spectral index between $4.9 \mathrm{GHz}$ and $8.5 \mathrm{GHz}$.

${ }^{\mathrm{f}} z_{\text {phot }}=0.34 \pm 0.04$ from SDSS DR9.

g Measured on the FIRST image; a flux density of $890 \pm 45 \mathrm{mJy}$ is measured on the A-array image (Figure 7(b)) and $960 \pm 48$ mJy on the C-array image (Figure 7(a)).

h $z_{\text {phot }}=0.57 \pm 0.08$ from SDSS DR9.

${ }^{\mathrm{i}}$ Data from Kale et al. (2013).

cluster minihalo (Bagchi et al. 2009) is very uncertain (Feretti et al. 2012).

Besides the radio properties of the minihalos, Table 5 provides values of radio power of the $\mathrm{BCG}$, cluster X-ray temperature, and total mass that will be used in Section 6 for the discussion of our results. $M_{500}$, the cluster mass within $R_{500}{ }^{9}$. It is estimated from the Sunyaev-Zeldovich (SZ) effect measured by Planck as described in Planck collaboration et al. (2013).

\section{NEW RADIO MINIHALOS}

Our reanalysis of the VLA data of the clusters A 478, RX J1532.9+3021, ZwCl 3146, and A 2204 led to the detection of large-scale diffuse emission in their cool cores, which

\footnotetext{
$9 R_{500}$, is the radius corresponding to a total density contrast $500 \rho_{c}(z)$, with $\rho_{c}(z)$ being the critical density of the universe at the cluster redshift.
}

we classify as minihalos. In the following sections, we describe these newly discovered minihalos and summarize their properties.

\subsection{A 478}

A 478 is a nearby $(z=0.09)$ cool-core cluster with a remarkably relaxed X-ray morphology and symmetric temperature distribution on large scales (e.g., Bourdin \& Mazzotta 2008). In the cool core, a high-resolution Chandra image shows significant substructure, resulting from the interaction of the central active radio galaxy with the surrounding ICM (Sun et al. 2003). Two small X-ray cavities, located within the central $r \sim 9^{\prime \prime}$ $(\sim 15 \mathrm{kpc})$, are partially filled by the radio galaxy lobes at $1.4 \mathrm{GHz}$. With a size of only $\sim 4 \mathrm{kpc}$, these are among the smallest cavities found in cluster cores.

VLA $1.4 \mathrm{GHz}$ images of A 478 at several resolutions are presented in Figure 1. The C-configuration image in panel (a) 
Table 5

Properties of Minihalos and Their Cluster Hosts

\begin{tabular}{|c|c|c|c|c|c|c|c|c|}
\hline Cluster Name & $\begin{array}{l}\mathrm{S}_{\mathrm{MH}, 1.4 \mathrm{GHz}} \\
(\mathrm{mJy})\end{array}$ & $\begin{array}{c}P_{\mathrm{MH}, 1.4 \mathrm{GHz}} \\
\left(10^{24} \mathrm{~W} \mathrm{~Hz}^{-1}\right)\end{array}$ & $\begin{array}{l}R_{\mathrm{MH}} \\
(\mathrm{kpc})\end{array}$ & $\begin{array}{c}P_{\mathrm{BCG}, 1.4 \mathrm{GHz}} \\
\left(10^{24} \mathrm{~W} \mathrm{~Hz}^{-1}\right)\end{array}$ & $\begin{array}{l}k T_{\mathrm{ncc}} \\
(\mathrm{keV})\end{array}$ & $\begin{array}{c}M_{500} \\
\left(10^{14} M_{\odot}\right)\end{array}$ & Ref. & Notes \\
\hline \multicolumn{9}{|l|}{ New detections } \\
\hline MACS J0159.8-0849 & $2.4 \pm 0.2$ & $1.40 \pm 0.14$ & 90 & $20.3 \pm 0.1$ & $9.36_{-0.67}^{+0.77}$ & $6.88_{-0.98}^{+0.90}$ & 1,5 & Candidate \\
\hline MACS J0329.6-0211 & $3.8 \pm 0.4$ & $2.84 \pm 0.30$ & $70^{\mathrm{a}}$ & $2.8 \pm 0.1$ & $6.44_{-0.45}^{+0.50}$ & $\ldots$ & 1,5 & Candidate \\
\hline A 478 & $16.6 \pm 3.0$ & $0.32 \pm 0.06$ & $160^{\mathrm{b}}$ & $0.60 \pm 0.03$ & $7.27_{-0.25}^{+0.26}$ & $7.06_{-0.36}^{+0.35}$ & 1,5 & \\
\hline ZwCl 3146 & $\sim 5.2^{\mathrm{c}}$ & $\sim 1.39$ & $90^{\mathrm{d}}$ & $\sim 0.8^{\mathrm{e}}$ & $7.46_{-0.30}^{+0.32}$ & $\ldots$ & 1,5 & \\
\hline A 1795 & $85.0 \pm 4.9$ & $0.79 \pm 0.05$ & $100^{\mathrm{f}}$ & $8.5 \pm 0.4$ & $6.05_{-0.15}^{+0.15}$ & $4.54_{-0.21}^{+0.21}$ & 1,5 & Candidate \\
\hline RX J1532.9+3021 & $7.5 \pm 0.4$ & $3.35 \pm 0.17$ & 100 & $7.0 \pm 0.4$ & $6.06_{-0.39}^{+0.43}$ & $\ldots$ & 1,5 & \\
\hline A 2204 & $8.6 \pm 0.9$ & $0.54 \pm 0.05$ & 50 & $3.7 \pm 0.2$ & $9.35_{-0.41}^{+0.43}$ & $7.96_{-0.38}^{+0.37}$ & 1,5 & \\
\hline $\mathrm{ZwCl} 1742.1+3306$ & $13.8 \pm 0.8$ & $0.20 \pm 0.01$ & 40 & $0.97 \pm 0.05$ & $5.23_{-0.73}^{+0.84}$ & $6.44_{-0.76}^{+0.71}$ & 1,6 & Uncertain $^{\mathrm{f}}$ \\
\hline MACS J1931.8-2634 & $47.9 \pm 2.8$ & $20.0 \pm 1.2$ & 100 & $4.9 \pm 0.2$ & $6.85_{-0.61}^{+0.73}$ & $6.19_{-0.83}^{+0.77}$ & 1,5 & Uncertain $^{\mathrm{g}}$ \\
\hline \multicolumn{9}{|c|}{ Previously known minihalos } \\
\hline Perseus & $3020 \pm 153$ & $2.18 \pm 0.11$ & 130 & $13.4 \pm 0.1^{\mathrm{h}}$ & $6.42_{-0.06}^{+0.06} *$ & $\ldots$ & 2,7 & \\
\hline A 1835 & $6.1 \pm 1.3$ & $1.19 \pm 0.25$ & $240^{\mathrm{i}}$ & $6.3 \pm 0.3$ & $9.77_{-0.51}^{+0.55}$ & $8.46_{-0.57}^{+0.55}$ & 3,5 & \\
\hline Ophiuchus & $83.4 \pm 6.6^{\mathrm{j}}$ & $0.15 \pm 0.02$ & 250 & $0.064 \pm 0.003$ & $10.25_{-0.22}^{+0.18} *$ & $\ldots$ & 3,7 & \\
\hline A 2029 & $19.5 \pm 2.5$ & $0.28 \pm 0.04$ & $270^{\mathrm{i}}$ & $7.4 \pm 0.4$ & $8.22_{-0.30}^{+0.31}$ & $6.82_{-0.25}^{+0.24}$ & 3,5 & \\
\hline A 2390 & $28.3 \pm 4.3$ & $4.46 \pm 0.67$ & $250^{\mathrm{i}}$ & $33.2 \pm 1.7$ & $10.85_{-0.31}^{+0.34}$ & $9.48_{-0.42}^{+0.41}$ & 3,5 & \\
\hline RBS 797 & $5.2 \pm 0.6^{\mathrm{k}}$ & $2.20 \pm 0.24$ & 120 & $7.6 \pm 0.4$ & $7.63_{-0.77}^{+0.94}$ & $6.27_{-0.66}^{+0.63}$ & 3,5 & \\
\hline RXC J1504.1-0248 & $20.0 \pm 1.0$ & $2.70 \pm 0.14$ & 140 & $5.7 \pm 0.3$ & $8.02_{-0.25}^{+0.26}$ & $6.98_{-0.60}^{+0.57}$ & 4,5 & \\
\hline RX J1347.5-1145 & $34.1 \pm 2.3$ & $25.59 \pm 1.79$ & 320 & $22.7 \pm 1.1$ & $15.12_{-0.86}^{+1.03}$ & $10.61_{-0.77}^{+0.74}$ & 3,5 & \\
\hline RX J1720.1+2638 & $72.0 \pm 4.4$ & $5.33 \pm 0.32$ & 140 & $0.50 \pm 0.02$ & $6.33_{-0.25}^{+0.29}$ & $6.34_{-0.40}^{+0.38}$ & 3,5 & \\
\hline MS $1455.0+2232$ & $8.5 \pm 1.1$ & $1.75 \pm 0.23$ & 120 & $0.96 \pm 0.05$ & $4.82_{-0.13}^{+0.14}$ & $\ldots$ & 3,5 & \\
\hline 2A $0335+096$ & $21.1 \pm 2.1^{\mathrm{k}}$ & $0.059 \pm 0.006$ & 70 & $0.058 \pm 0.003$ & $3.53_{-0.13}^{+0.10}$ & $2.27_{-0.25}^{+0.24}$ & 3,8 & \\
\hline A 2626 & $18.0 \pm 1.8$ & $0.14 \pm 0.01$ & 30 & $0.129 \pm 0.006$ & $3.29 *$ & $\ldots$ & 9,10 & Uncertain $^{1}$ \\
\hline
\end{tabular}

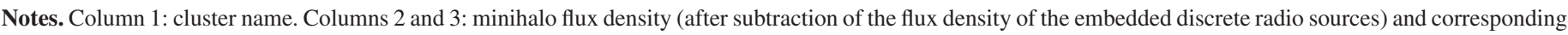

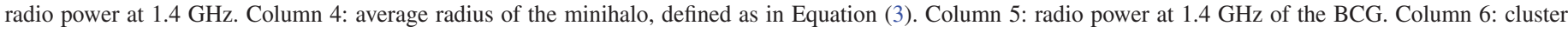

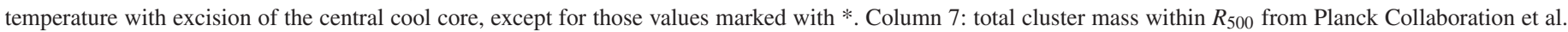
(2013). Column 8: references to radio flux density and cluster temperature.

a The minihalo may be more extended (see grayscale image in Figure 6(a)).

${ }^{b}$ Uncertain due to the presence of the HT source S4 (Figure 1(c)).

${ }^{c}$ Estimated from the NVSS image, after subtraction of the expected flux densities of the embedded radio galaxies (see Section 4.3).

${ }^{\mathrm{d}}$ Measured on the $4.9 \mathrm{GHz}$ image.

e Expected value, based on the source spectral index between $4.9 \mathrm{GHz}$ and $8.5 \mathrm{GHz}$.

$\mathrm{f}$ The minihalo may be more extended (see grayscale image in Figure 7(a)).

g The classification as a minihalo is uncertain (see Section 6.5).

h From NVSS.

${ }^{\mathrm{i}}$ Uncertain due to the presence of many discrete radio galaxies in the area covered by the minihalo emission.

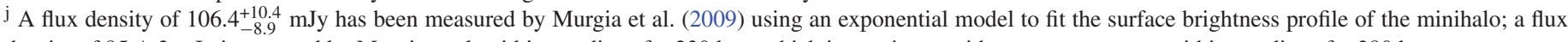
density of $85 \pm 3 \mathrm{mJy}$ is reported by Murgia et al. within a radius of $\sim 230 \mathrm{kpc}$, which is consistent with our measurement within a radius of $\sim 280 \mathrm{kpc}$.

$\mathrm{k}$ The contribution of the emission associated with the X-ray cavities has been subtracted out.

${ }^{1}$ The extended emission seen in the core (Gitti 2013, and references therein) may be associated with the central radio galaxy rather than being a minihalo.

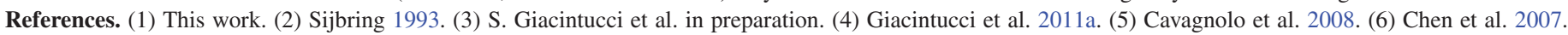
(7) Ikebe et al. 2002. (8) Hudson et al. 2010. (9) Gitti 2013. (10) Cavagnolo et al. 2009.

reveals the presence of a diffuse, low surface brightness radio source, which we classify as a minihalo. The minihalo encompasses the central bright radio galaxy (S1), unresolved at this resolution $\left(\mathrm{FWHM}=15^{\prime \prime}\right)$. At higher resolution, $\mathrm{S} 1$ has a double-lobe morphology with a size of $\sim 13 \mathrm{kpc}$, as seen in Figure 1(b), which shows the A-array image (white contours) overlaid on the Hubble Space Telescope (HST) Wide-Field Planetary Camera 2 (WFPC2) image (grayscale). We also report in black the C-array contours of S1 from Figure 1(a) to highlight the small size of the radio galaxy compared to the much larger scale of the surrounding minihalo.

East of the minihalo, a head-tail source (S4), which is a cluster member (Table 4 ), extends for $\sim 200 \mathrm{kpc}$, encom- passing the unresolved source S2. This latter is another cluster member (Table 4) and possesses a tailed morphology at arcsecond resolution (image not shown here), with both jets bent toward the northeast, i.e., almost perpendicular to the tail of S4. The unresolved source S3, north of S2 and S4, has no optical identification and is most likely a background source.

We produced a low angular resolution image to highlight the extended emission associated with the minihalo. The image is shown in Figure 1(c), overlaid on the Chandra image. The minihalo appears more extended toward the northeast ( $R_{\max } \sim 180 \mathrm{kpc}$ ), following a similar elongation of the X-ray surface brightness; its extent is $R_{\min } \sim 150 \mathrm{kpc}$ in the other 

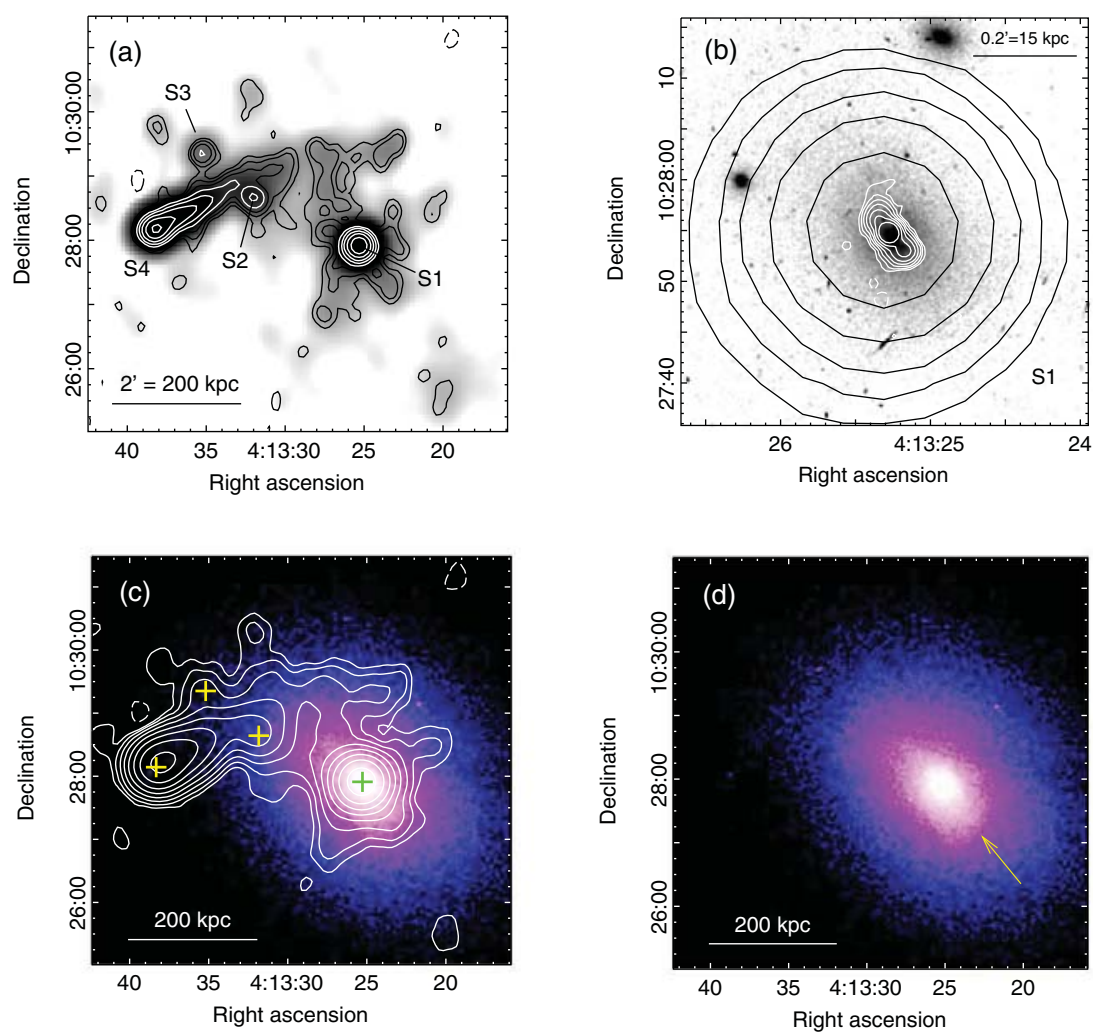

Figure 1. A 478. In all images, radio contours are -1 (dashed), $1,2,4,8,16, \ldots \times 3 \sigma$. (a) VLA $1.4 \mathrm{GHz}$ C-array image (white and black contours), restored with a $15^{\prime \prime}$ circular beam. The rms noise is $1 \sigma=40 \mu \mathrm{Jy}$ beam ${ }^{-1}$. The image is also shown in grayscale, smoothed with a Gaussian with a radius of 5 pixels $\left(20^{\prime \prime}\right)$. The individuals radio galaxies, listed in Table 4, are labeled. (b) VLA $1.4 \mathrm{GHz}$ A-array contours of S1 (white), restored with a 1" circular beam, overlaid on the optical HST WFPC2 image (grayscale). The rms noise is $1 \sigma=25 \mu \mathrm{Jy}$ beam $^{-1}$. The C-array contours of S1 are reported in black (the same as white contours in panel (a)). (c) Low-resolution VLA C-array contours at $1.4 \mathrm{GHz}$ of the minihalo and radio galaxies (green and yellow crosses), overlaid on the Chandra image in the $0.5-4 \mathrm{keV}$ band. The radio image has been restored with a $30^{\prime \prime}$ circular beam. The rms noise is $1 \sigma=50 \mu \mathrm{Jy} \mathrm{beam}^{-1}$. (d) Chandra image, the same as in (c). The arrow indicates the position of a cold front.

(A color version of this figure is available in the online journal.)

directions. However, to the NE, the minihalo emission partially blends with the tail of S4, and it is difficult to determine the boundaries of the two structures.

We measured the flux densities of S1, S2, and S3 on the A configuration image; consistent values were measured at lower resolutions. The emission associated with the extended source $\mathrm{S} 4$ was measured on the $\mathrm{C}$ configuration image (Figure 1(a)). $\mathrm{S} 1, \mathrm{~S} 2$, and $\mathrm{S} 4$ are also detected at $4.9 \mathrm{GHz}$ (Table 2; image not shown here). All flux densities and spectral indices are summarized in Table 4.

We subtracted the contribution of the radio galaxies S1-S4 from the total emission in Figure 1(c) and measured a flux density of $16.6 \pm 3.0 \mathrm{mJy}$ for the minihalo, where the large error ( 18\%; Equation (1)) reflects the difficulty of subtracting the head-tail S4. The corresponding radio power is $P_{1.4 \mathrm{GHz}}=$ $(3.2 \pm 0.6) \times 10^{23} \mathrm{~W} \mathrm{~Hz}^{-1}$.

A cold front has been detected in the Chandra image at $\sim 60 \mathrm{kpc}$ southwest of the center (Markevitch et al. 2003). Its position, marked by the arrow in Figure 1(d), is roughly coincident with the southwestern boundary of the minihalo, suggesting that the diffuse radio emission may be confined here by the front, as seen in other minihalos (Mazzotta \& Giacintucci 2008; ZuHone et al. 2013; S. Giacintucci et al., in preparation).

\section{2. $R X J 1532.9+3021$}

$\mathrm{RX} \mathrm{J1532.9+3021} \mathrm{is} \mathrm{an} \mathrm{X-ray} \mathrm{luminous} \mathrm{cluster} \mathrm{at} z=0.362$. It has a mean temperature of $\sim 6 \mathrm{keV}$ (Table 5) and is one of the most luminous cool cores known (Hlavacek-Larrondo et al. 2013). Recent Chandra data confirmed the presence of an $\mathrm{X}$-ray cavity west of the cluster center and revealed a second, less prominent, cavity on the opposite side. A cold front was also reported at a radius of $65 \mathrm{kpc}$, partially coincident with the edge of the western cavity (Hlavacek-Larrondo et al. 2013; see also Figure 2(f)).

The VLA observations analyzed in this article reveal a prominent minihalo in the cluster core. Figure 2(a) shows the $1.4 \mathrm{GHz}$ image of the minihalo from the combined $\mathrm{A}+\mathrm{B}$ array data set $\left(\sim 3^{\prime \prime}\right.$ resolution). The diffuse source is relatively round in shape, with a radius of $\sim 100 \mathrm{kpc}$, and totally encloses the central unresolved source S1 associated with the BCG. Figure 2(b) shows an optical HST image of the BCG with an overlay of the $1.4 \mathrm{GHz}$ A-configuration contours of the source S1 (unresolved) and some of the surrounding diffuse emission associated with the minihalo.

We also obtained images from the archival VLA observations at $325 \mathrm{MHz}$ in A configuration and at $4.9 \mathrm{GHz}$ in $\mathrm{C}$ configuration (shown in Figures 2(c) and (d)), as well as at $8.5 \mathrm{GHz}$ (A and D configurations; not shown) and $22 \mathrm{GHz}$ (D configuration; not shown). Details of all these observations are given in Table 2. The $325 \mathrm{MHz}$ image clearly shows diffuse emission around S1 on a spatial scale that is similar to the extent of the minihalo at $1.4 \mathrm{GHz}$ (overlaid in magenta). At $4.9 \mathrm{GHz}$, only the innermost and brightest portion of the minihalo is detected. The minihalo is not detected in the images at higher frequencies where only the source S1 is detected. This source is still unresolved at the 

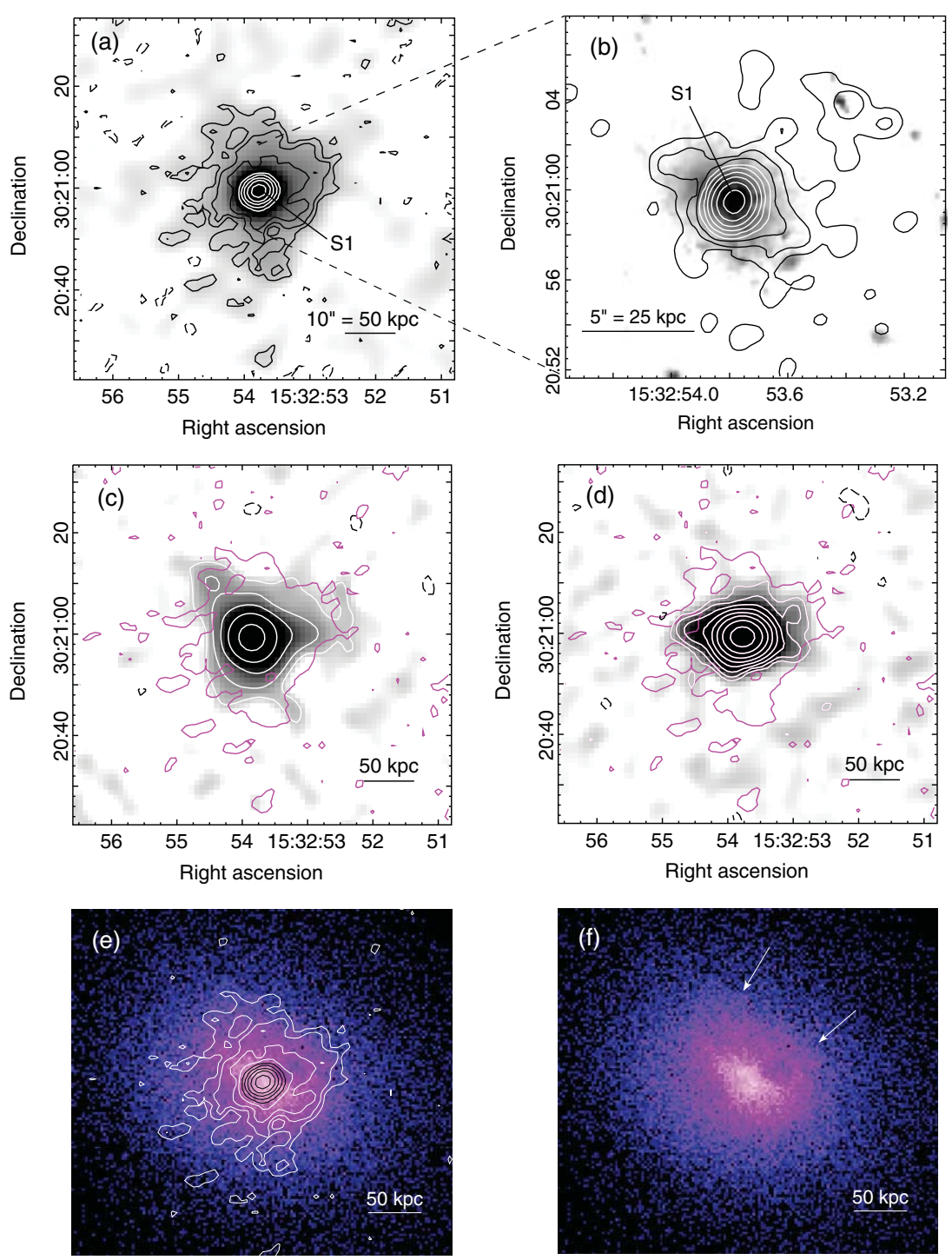

Figure 2. RX J1532.9+3021. In all images, radio contours are -1 (dashed), $1,2,4,8,16, \ldots \times 3 \sigma$. (a) VLA $1.4 \mathrm{GHz}$ contours (black and white) from the combined $\mathrm{A}+\mathrm{B}$ array. The beam is $3^{\prime \prime} .4 \times 2^{\prime \prime} .9$ in p.a. $-65^{\circ}$ and $1 \sigma=10 \mu \mathrm{Jy}$ beam ${ }^{-1}$. The image is also shown in grayscale, smoothed with a Gaussian with a radius of 4 pixels $\left(3^{\prime \prime}\right)$. S1 is the central radio galaxy (see panel (b) and Table 4). (b) VLA A-array $1.4 \mathrm{GHz}$ contours (black and white), overlaid on the HST WFPC2 image (grayscale). The beam is $1^{\prime \prime} .4 \times 1^{\prime \prime} .1 \mathrm{in} \mathrm{p.a.}-29^{\circ}$ and $1 \sigma=12 \mu \mathrm{Jy}$ beam ${ }^{-1}$. (c) VLA A-array image at $325 \mathrm{MHz}$ (grayscale and white contours). The beam is $7^{\prime \prime} .0 \times 6{ }^{\prime \prime} .5$ in p.a. $-37^{\circ}$ and $1 \sigma=400 \mu \mathrm{Jy}_{\text {beam }}{ }^{-1}$. The lowest contour at $1.4 \mathrm{GHz}$ from panel (a) is shown in magenta. (d) VLA C-array image at $4.9 \mathrm{GHz}$ (grayscale and white contours). The beam is $6{ }^{\prime \prime} 0 \times 4^{\prime \prime} .9$ in p.a., $90^{\circ}$ and $1 \sigma=15 \mu \mathrm{Jy}$ beam ${ }^{-1}$. The lowest contour at $1.4 \mathrm{GHz}$ from panel (a) is shown in magenta. (e) VLA $1.4 \mathrm{GHz}$ contours (the same as (a)), overlaid on the Chandra image in the $0.5-7 \mathrm{keV}$ band (from Hlavacek-Larrondo et al. 2013). The image has the same physical size as panel (a). (f) Chandra image, the same as in (e). Arrows indicate the position of a cold front. A prominent cavity is also visible west of the cluster center (see Hlavacek-Larrondo et al. 2013). (A color version of this figure is available in the online journal.)

resolution of 0.2 of the $8.5 \mathrm{GHz}$ A-array image, which implies that its linear size $<1 \mathrm{kpc}$.

The detection of a minihalo in this cluster was also reported by Hlavacek-Larrondo et al. (2013), who presented similar VLA images at $1.4 \mathrm{GHz}$ and $325 \mathrm{MHz}$ from the same data sets and a spectral index image between these two frequencies. The cluster was also observed with the GMRT at $610 \mathrm{MHz}$ as part of the GRHS. A reanalysis of the $610 \mathrm{MHz}$ observations, aimed to image the newly discovered minihalo, is presented by Kale et al. (2013). The morphology of the diffuse source at $610 \mathrm{MHz}$ is similar in shape and size to the minihalo structure seen at $1.4 \mathrm{GHz}$ (see their Figure 2).

The flux densities measured for $\mathrm{S} 1$ at all frequencies, including the $610 \mathrm{MHz}$ value from Kale et al. (2013), are reported in Table 1. The source has a spectral index $\alpha=0.43 \pm 0.02$ between
$325 \mathrm{MHz}$ and $22 \mathrm{GHz}$. After the subtraction of S1, the minihalo has a flux density of $7.5 \pm 0.4 \mathrm{mJy}$ at $1.4 \mathrm{GHz}$, which corresponds to a radio power of $(3.4 \pm 0.2) \times 10^{24} \mathrm{~W} \mathrm{~Hz}^{-1}$ (Table 5). Its flux density is $33.5 \pm 4.4 \mathrm{mJy}$ at $325 \mathrm{MHz}, 16.0 \pm 0.8 \mathrm{mJy}$ at $610 \mathrm{MHz}$, and $1.3 \pm 0.2 \mathrm{mJy}$ at $4.9 \mathrm{GHz}$ (all excluding S1). The integrated spectrum of the minihalo, based on these measurements, is shown in Figure 12 and discussed in Section 6.3. The total spectral index is $\alpha=1.20 \pm 0.07$.

The minihalo image is overlaid on the Chandra image in Figure 2(e). The position of the cold front is indicated by the arrows in panel (f). As noticed by Hlavacek-Larrondo et al. (2013), the minihalo emission is apparently contained within the region delineated by the front. Part of the extended emission is spatially coincident with a prominent X-ray cavity to the west of S1 (also visible in panel (f)) and with a second, weaker 

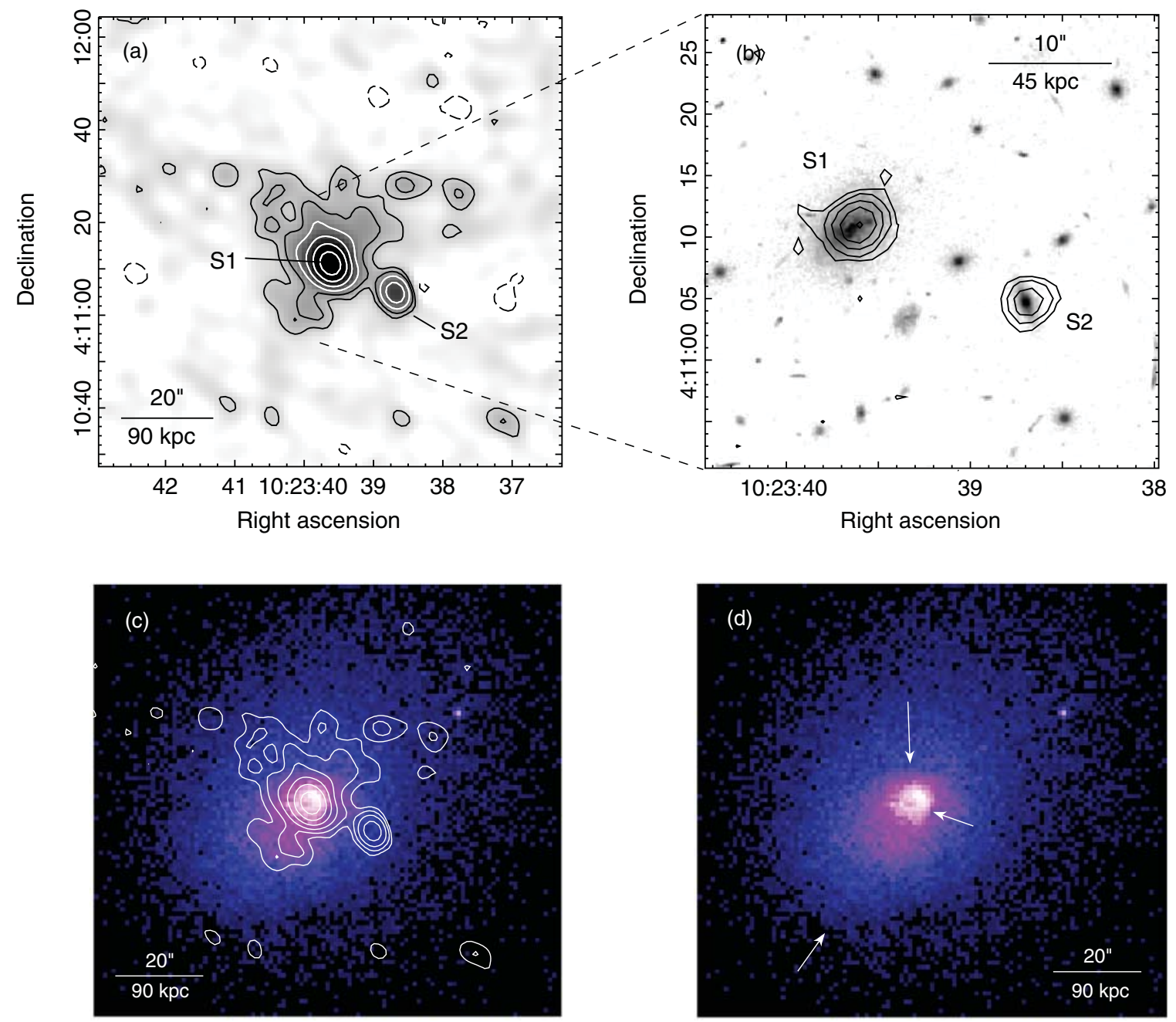

Figure 3. ZwCl 3146. (a) VLA C-array contours at $4.9 \mathrm{GHz}$ (black and white) at the resolution of $5^{\prime \prime} .7 \times 44^{\prime \prime} 6$, in p.a. $27^{\circ}$. The rms noise is $1 \sigma=10 \mu \mathrm{Jy}$ beam ${ }^{-1}$. Contours are -1 (dashed) $1,2,4,8,16, \ldots \times 3 \sigma$. The radio galaxies are labeled (see panel(b) and Table 4). The image is also shown in grayscale, smoothed with a Gaussian with a radius of 3 pixels ( $\left.3^{\prime \prime}\right)$. (b) VLA C-array contours at $8.5 \mathrm{GHz}$, overlaid on the HST WFPC2 image (grayscale). The restoring beam is $2^{\prime \prime}$. 5 2 2". 4 in p.a. $-34^{\circ}$. The rms noise is $1 \sigma=15 \mu \mathrm{Jy}$ beam $^{-1}$. Contours are $1,2,4,8,16, \ldots \times 3 \sigma$. No levels at $-3 \sigma$ are present in the portion of the image shown. (c) $4.9 \mathrm{GHz}$ radio contours (the same as in (a)) overlaid on the Chandra image in the $0.4-5 \mathrm{keV}$ band. The image has the same physical scale as panel (a). (d) Chandra image, the same as in (c). Arrows show the position of the cold fronts.

(A color version of this figure is available in the online journal.)

cavity, which has been recently found by Hlavacek-Larrondo et al. (2013). This indicates that a pair of radio lobes may be superposed to the larger-scale minihalo emission (see also Hlavacek-Larrondo et al. 2013).

In the field of RX J1532.9+3021, we detected a complex radio source, possibly associated with a nearby galaxy cluster. The radio images and description of this source are given in the Appendix.

\section{3. $\mathrm{ZwCl} 3146$}

ZwCl 3146 is a hot $(k T \sim 7.5 \mathrm{keV}$; Table 5) and relaxed cluster at $z=0.29$. It exhibits a pronounced cool core with one of the strongest cooling rates known $\left(\sim 1600 M_{\odot} \mathrm{yr}^{-1}\right.$; Kausch et al. 2007). Multiple cold fronts have been detected by Chandra in its core (Forman et al. 2002); two of them are visible at small radii ( $\sim 3^{\prime \prime}$ and $8^{\prime \prime}$, corresponding to $\sim 13 \mathrm{kpc}$ and $\sim 35 \mathrm{kpc}$ ), while the third front is located at $\sim 35^{\prime \prime}(\sim 150 \mathrm{kpc})$ from the cluster center (see also Figure 3(d)).
No pointed VLA observations at $1.4 \mathrm{GHz}$ exist for $\mathrm{ZwCl} 3146$. Inspection of the $1.4 \mathrm{GHz}$ image from the NVSS ${ }^{10}$ indicates that the cluster hosts a central radio source, whose structure is unresolved at the $45^{\prime \prime}$ resolution of the image. A Gaussian fit to the source gives an integrated flux density of $8.7 \pm 0.5 \mathrm{mJy}$.

We used VLA observations at $4.9 \mathrm{GHz}$ and $8.5 \mathrm{GHz}$ to image the radio source at higher resolutions (Table 2). A central point source, embedded in faint, extended emission, is detected in the $4.9 \mathrm{GHz} \sim 5^{\prime \prime}$ resolution image (Figure 3(a)). The diffuse, amorphous feature, which we classify as a minihalo, has a radius of $\sim 90 \mathrm{kpc}$. A second point source (S2) is detected southwest of $\mathrm{S} 1$. The $2^{\prime \prime}$ resolution image at $8.5 \mathrm{GHz}$ is shown in Figure 3(b), overlaid on the optical HST image. Both point sources have optical counterparts: S1 is identified with the BCG, and S2 is associated with a background galaxy with a photometric redshift $z_{\text {phot }}=0.34$ from the SDSS (Table 4). No extended emission connected to these sources, such as jets or lobes, is detected. The

\footnotetext{
10 NRAO VLA Sky Survey, Condon et al. (1998).
} 

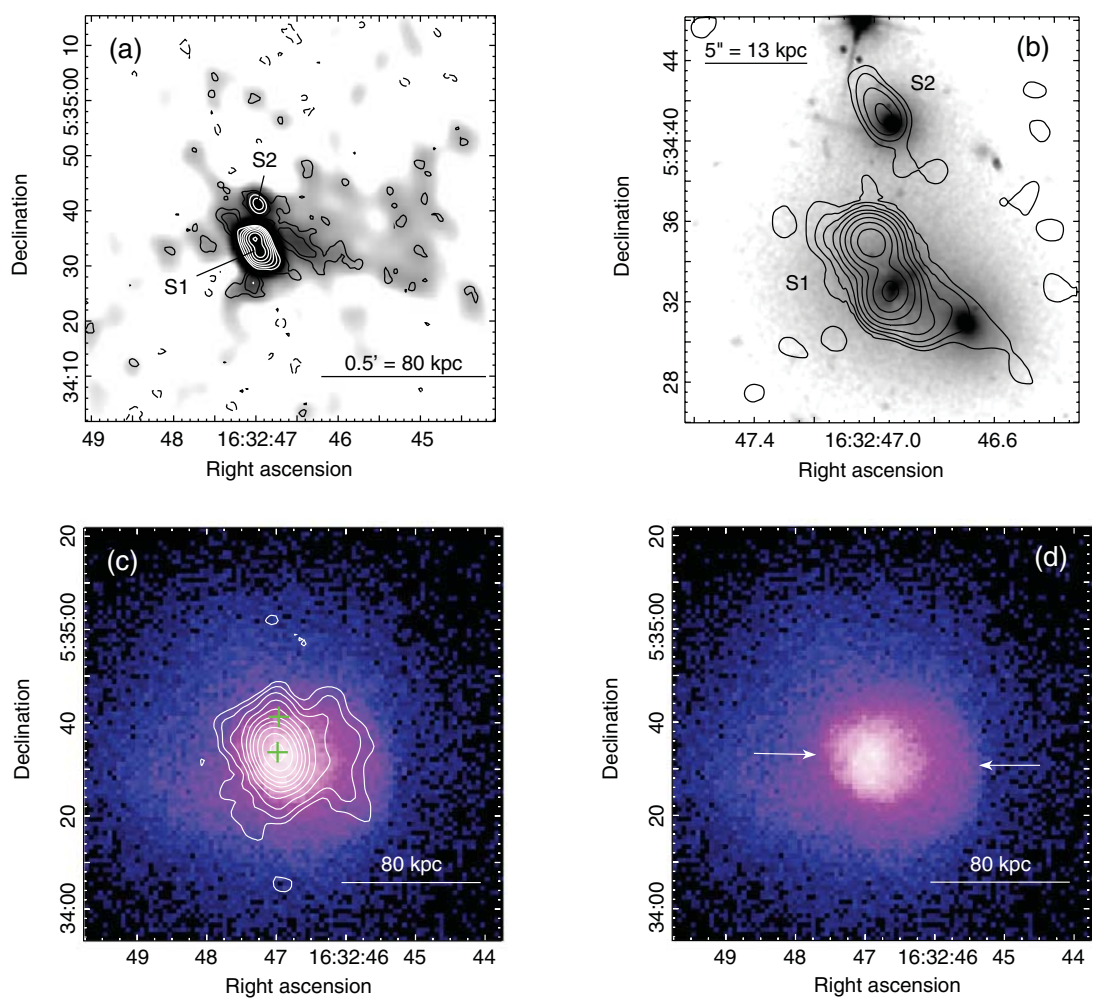

Figure 4. A 2204. In all images, radio contours are -1 (dashed), $1,2,4,8,16, \ldots \times 3 \sigma$. (a) VLA $1.4 \mathrm{GHz}$ contours (black and white) from the combined A+B array. The restoring beam is $2^{\prime \prime} .0 \times 1^{\prime \prime} .9$ in p.a. $77^{\circ}$, and the rms noise is $1 \sigma=30 \mu \mathrm{Jy}$ beam ${ }^{-1}$. The image is also shown in grayscale, smoothed with a Gaussian with a radius of 5 pixels $\left(2^{\prime \prime}\right)$. S1 is the central radio galaxy, and S2 is associated with a nearby galaxy (see panel (b) and Table 4). (b) VLA-A 1.4 GHz contours of S1 and S2, overlaid on the HST WFPC2 image (grayscale). The restoring beam is $1.4 \times 1.3$ in p.a. $45^{\circ}$, and rms noise is $1 \sigma=25 \mu \mathrm{Jy}_{\text {beam }}^{-1}$. No levels at $-3 \sigma$ are present in the portion of the image shown. (c) VLA-B contours at $1.4 \mathrm{GHz}$ of the minihalo and discrete radio sources (green crosses), overlaid on the Chandra image in the $0.5-4 \mathrm{keV}$ band. The restoring beam is $6{ }^{\prime \prime} 0 \times 5^{\prime \prime} 0$ in p.a., $17^{\circ}$, and the rms noise is $1 \sigma=30 \mu \mathrm{Jy}$ beam $^{-1}$. No levels at $-3 \sigma$ are present in the portion of the image shown. (d) Chandra image, the same as in (c). Arrows indicate the position of two cold fronts.

(A color version of this figure is available in the online journal.)

flux densities of S1 and S2 are summarized in Table 4. Their spectral indices are $\alpha=0.66 \pm 0.14$ (S1) and $\alpha=-0.33 \pm 0.16$ (S2). After subtraction of the flux densities of S1 and S2 from the total emission in Figure 3(a), a flux of $1.7 \pm 0.2 \mathrm{mJy}$ is found for the minihalo at $4.9 \mathrm{GHz}$. Assuming a power-law spectrum extrapolated from the $4.9-8.5 \mathrm{GHz}$ frequencies, the expected flux densities of $\mathrm{S} 1$ and $\mathrm{S} 2$ at $1.4 \mathrm{GHz}$ are $3.3 \mathrm{mJy}$ and $0.2 \mathrm{mJy}$, respectively. Subtraction of these values from the total flux density in the NVSS image provides an estimate of the flux density of the minihalo at $1.4 \mathrm{GHz}$ of $\sim 5.2 \mathrm{mJy}$. The corresponding radio power is $\sim 1.4 \times 10^{24} \mathrm{~W} \mathrm{~Hz}^{-1}$, and the estimated 1.4-4.9 GHz spectral index of the minihalo is $\alpha \sim 1$.

The minihalo is overlaid on the Chandra image in Figure 3(c), which is also shown in panel (d) with arrows marking the position of the cold fronts. The minihalo emission extends mostly in the northern and SE sectors of the core. No clear radio features are visible at the position of the cold fronts: the innermost front lies inside the minihalo, near the central point source; the outer fronts are at larger radii than the minihalo.

Because of their steep radio spectra $(\alpha \sim 1.2-1.3)$ and low surface brightness, minihalos are usually best detected at frequencies around $1 \mathrm{GHz}$ or lower. There are very few detections at higher frequencies, e.g., 2A 0335+096 (Sarazin et al. 1995; S. Giacintucci et al., in preparation), RXCJ $1720.1+26$ (S. Giacintucci et al., in preparation) and RX J1532.9+3021 (see this article). It is therefore noteworthy that this minihalo has been discovered at $4.9 \mathrm{GHz}$.

\subsection{A 2204}

A2204 is a $\sim 9 \mathrm{keV}$ cluster (Table 5) at $z=0.152$, regular and relaxed on large scales but disturbed in its cool core where metallicity substructures and multiple X-ray cavities have been revealed by Chandra (Sanders et al. 2005, 2009). Chandra also showed a clear spiral structure in the core surface brightness distribution, defined by two cold fronts at $\sim 28 \mathrm{kpc}$ and $\sim 55 \mathrm{kpc}$ from the center (Sanders et al. 2005; ZuHone et al. 2010, see also Figure 4(d)). A small radio source is associated with the BCG (Sanders et al. 2009). At arcsecond resolution, it consists of a compact, flat-spectrum core and two weak, extended components to the north and south. Hints of further extended emission to the east and west of the central radio galaxy were reported by Sanders et al. (2005, 2009), who suggested the possible existence of a larger-scale minihalo.

We reprocessed the VLA data at $1.4 \mathrm{GHz}$ analyzed by Sanders et al. (2009). Our radio images are presented in Figure 4. In the images from the $\mathrm{A}$ and combined $\mathrm{A}+\mathrm{B}$ configurations (panels (a) and (b)), we detect the central cluster radio galaxy (S1) and a second unresolved source (S2), associated with a galaxy without redshift information. Their morphology and flux densities (Table 4) are consistent with those reported by Sanders et al. (2009). The smoothed grayscale image in panel (a) shows the diffuse emission west of S1, first noticed by Sanders et al. (2005). To investigate this component, we produced an image from the B array applying natural weighting. The image is overlaid on the Chandra image in Figure 4(c). Diffuse radio emission is clearly present in the area around S1 and S2 

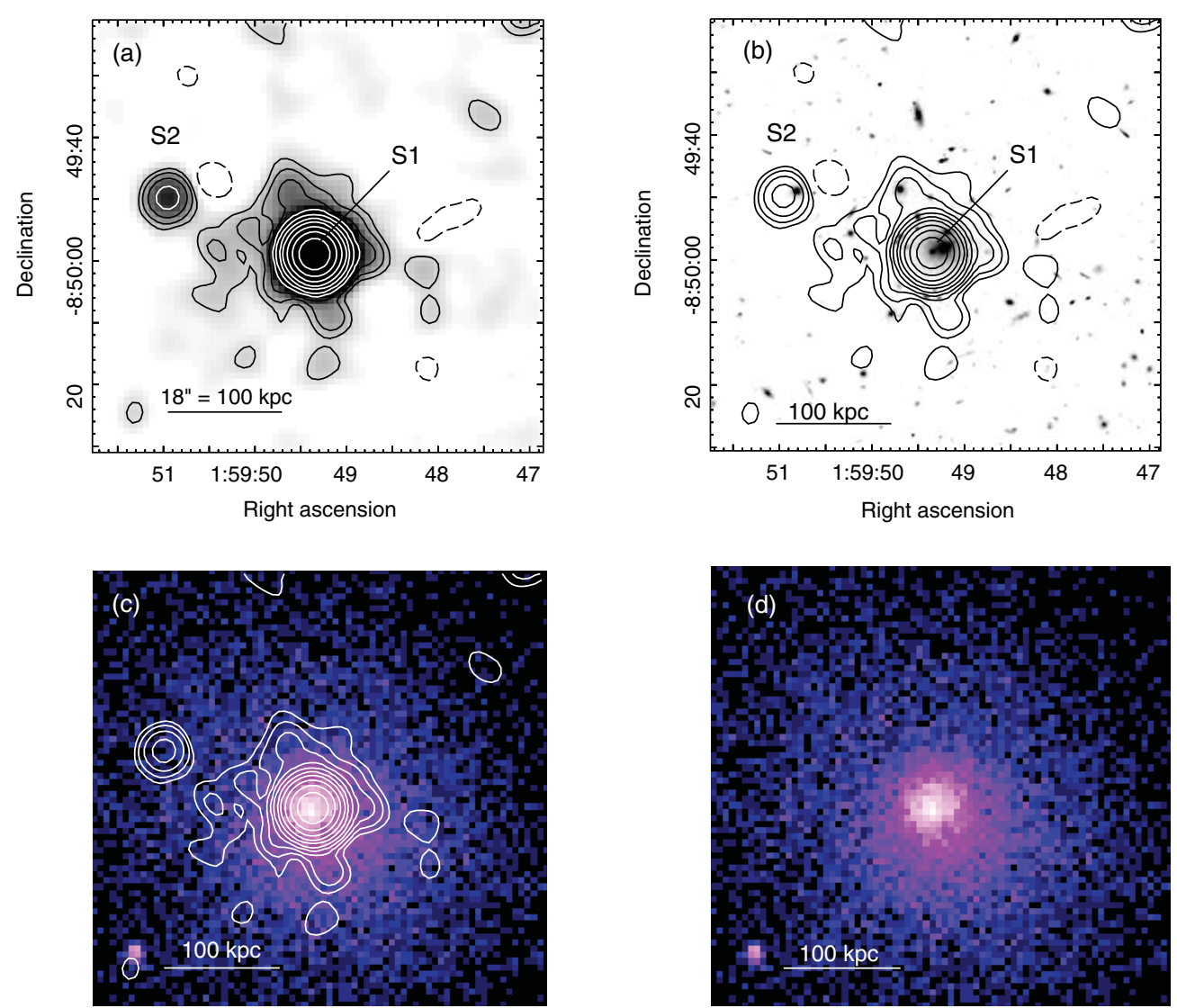

Figure 5. MACS J0159.8-0849. (a) VLA B-array contours at $1.4 \mathrm{GHz}$ (black and white), restored with a $5^{\prime \prime}$ circular beam. The rms noise is $1 \sigma=15 \mu \mathrm{Jy}$ beam ${ }^{-1}$, and contours are -1 (dashed), $1,2,4,8,16, \ldots \times 3 \sigma$. The image is also shown in grayscale, smoothed with a Gaussian with a radius of 3 pixels $\left(3^{\prime \prime}\right)$. The unresolved source S1 is associated with the BCG, and S2 is a possible cluster member (see panel (b) and Table 4). (b) $1.4 \mathrm{GHz}$ contours, the same as in (a), overlaid on the HST WFPC2 image (grayscale). (c) $1.4 \mathrm{GHz}$ contours, the same as in (a), overlaid on the Chandra image in the $0.5-4 \mathrm{keV}$ band. The image has the same physical size as panel (a). (d) Chandra image, the same as in (c).

(A color version of this figure is available in the online journal.)

(crosses), confirming the initial suggestion of Sanders et al. (2005) of a centrally located minihalo. The source is $\sim 50 \mathrm{kpc}$ in radius and has a total flux density of $8.6 \pm 0.9 \mathrm{mJy}$ (after subtraction of S1 and S2), which corresponds to a radio power of $(5.4 \pm 0.5) \times 10^{23} \mathrm{~W} \mathrm{~Hz}^{-1}$.

In Figure 4(d), arrows mark the position of the two cold fronts that define the prominent spiral pattern in the Chandra image. The minihalo emission appears to be contained within the region bounded by the fronts, as observed in other minihalo clusters with similar spiral-like patterns in their cool cores (Mazzotta \& Giacintucci 2008; ZuHone et al. 2013).

\section{CANDIDATE MINIHALOS}

In six of the clusters listed in Table 1, we found diffuse radio emission around the dominant radio galaxies; but classifications of these clusters as minihalos are uncertain and require further observations. The radio images of these new candidate minihalos are discussed here.

\subsection{MACS J0159.8-0849}

MACS J0159.8-0849 is a relatively high redshift $(z=0.405)$ hot ( $\sim 9 \mathrm{keV}$; Table 5) cluster with regular X-ray morphology (e.g., Maughan et al. 2008). The X-ray surface brightness profile peaks at the BCG, and the gas temperature declines toward the center (e.g., Cavagnolo et al. 2009), as typically observed in other cool-core clusters.
We imaged the central region of the cluster at $1.4 \mathrm{GHz}$ using VLA observations in B configuration (Table 2). Our image is shown in Figure 5, where it is also overlaid on the HST optical and Chandra X-ray images. We detected a point source (S1) at the BCG and a second unresolved source $\sim 0{ }^{\prime} 4$ to the NE, both associated with a possible cluster member galaxy with $z_{\text {phot }}=0.44$ (Table 4 ). We also detect a $\sim 90 \mathrm{kpc}$ radius diffuse source around $\mathrm{S} 1$, which is probably a minihalo. To resolve $\mathrm{S} 1$ and investigate its connection with the larger-scale extended emission, we checked the VLA archive for higher-resolution observations. There is a pointed observation at $8.5 \mathrm{GHz}$ in A configuration, which we used to obtain an image of the BCG at subarcsecond resolution (not shown here). We found a bright point source coincident with the peak of $\mathrm{S} 1$ in the $1.4 \mathrm{GHz}$ image. No other radio emission is detected at the sensitivity of the $8.5 \mathrm{GHz}$ image $\left(1 \sigma=18 \mu \mathrm{Jy}\right.$ beam $\left.^{-1}\right)$.

We measured the flux density of $\mathrm{S} 1$ at $1.4 \mathrm{GHz}$ on an image obtained excluding the innermost $15 \mathrm{k} \lambda$ region of the $u-v$ plane, which is sensitive to the larger-scale emission, and found $36.7 \pm 1.8 \mathrm{mJy}$. Comparison between the flux densities at $1.4 \mathrm{GHz}$ and $8.5 \mathrm{GHz}(94.8 \pm 4.8 \mathrm{mJy})$ gives $\alpha=-0.55 \pm 0.04$, suggesting that $\mathrm{S} 1$ is likely dominated by a bright core with an inverted spectrum. To estimate the flux density of the candidate minihalo at $1.4 \mathrm{GHz}$, we subtracted the flux density of S1 and $\mathrm{S} 2$ from the total emission measured in Figure 5(a), $37.8 \mathrm{mJy}$. We found $2.4 \pm 0.2 \mathrm{mJy}$ in diffuse emission, which corresponds to a radio power of $(1.40 \pm 0.14) \times 10^{24} \mathrm{~W} \mathrm{~Hz}^{-1}$. 

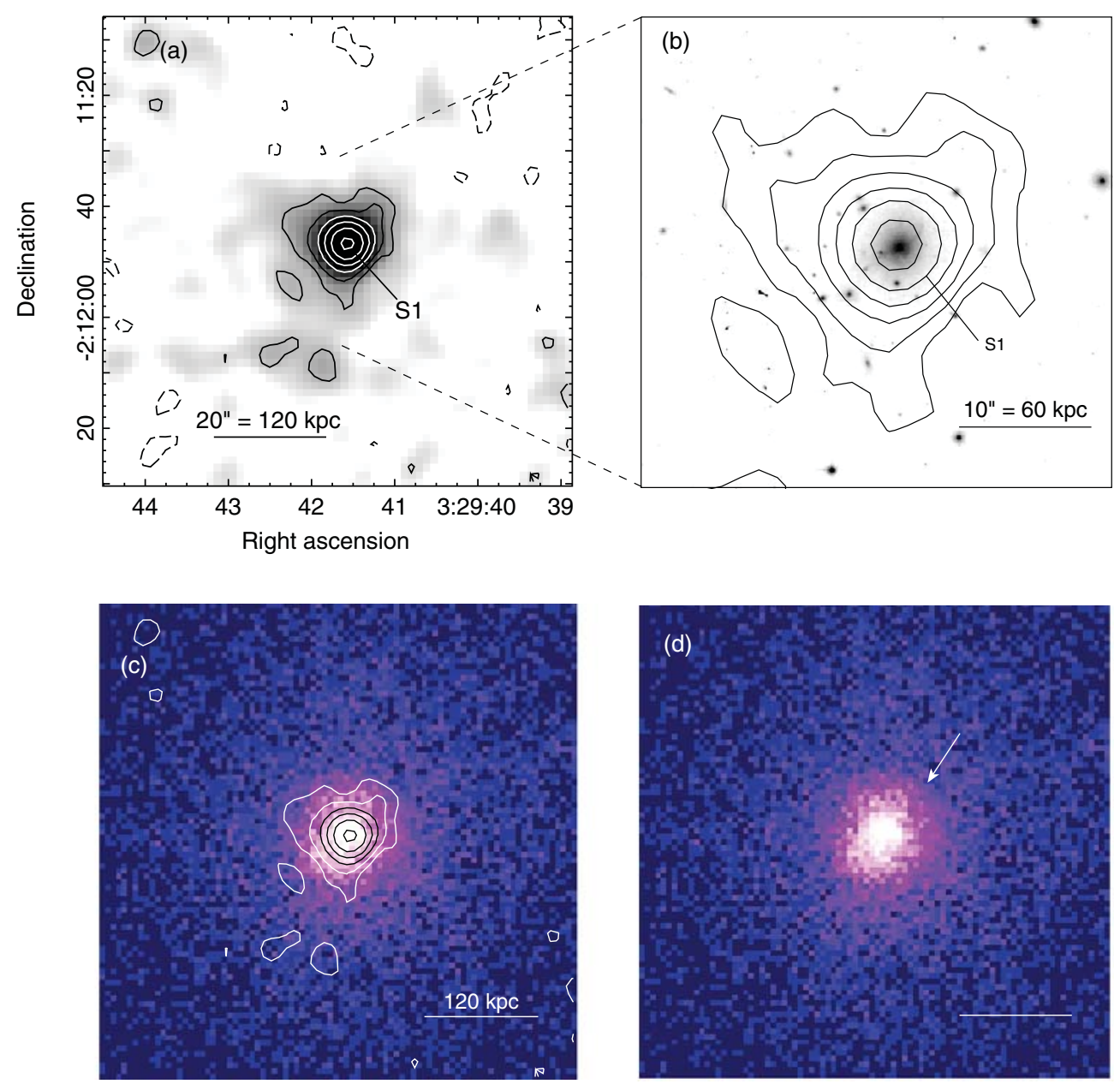

Figure 6. MACS J0329.6-0214. In all images, radio contours are -1 (dashed), $1,2,4,8,16, \ldots \times 3 \sigma$. (a) VLA 1.4 GHz contours (black and white) at the resolution of 4."7 × 4".5 in p.a. $0^{\circ}$ (image credit: NRAO/VLA Archive Survey, (c) 2005-2007 AUI/NRAO). The rms noise is $1 \sigma=40 \mu \mathrm{Jy}^{\text {beam }}{ }^{-1}$. The image is also shown in grayscale, smoothed with a Gaussian with a radius of 3 pixels (4"). The source S1 is associated with the BCG (see panel (b) and Table 4). (b) 1.4 GHz contours (the same as in (a)), overlaid on the HST ACS image (grayscale). (c) $1.4 \mathrm{GHz}$ contours (black and white, the same as in (a)), overlaid on the Chandra image in the $0.5-4 \mathrm{keV}$ band. The image has the same physical scale as panel (a). (d) Chandra image, the same as in (c). The arrow indicates a surface brightness edge at $\sim 10^{\prime \prime}$ from the center, which may be a cold front.

(A color version of this figure is available in the online journal.)

The comparison between the radio and X-ray Chandra images is shown in Figure 5(c). The diffuse radio emission permeates most of the core region and has a similar roundish distribution. No obvious features are seen in the X-ray surface brightness.

The confirmation of the minihalo nature of the central extended emission requires further observations. In particular, higher resolution images are needed to determine the morphology of the central radio galaxy and whether it has any connection to the surrounding emission. MACS J0159.8-0849 has recently been observed with the GMRT at $325 \mathrm{MHz}$ as part of a cluster survey (G. Macario et al., in preparation). Diffuse emission is visible in the $325 \mathrm{MHz}$ image on a similar spatial scale. However, the angular resolution of the GMRT at this frequency $\left(\sim 10^{\prime \prime}\right)$ does not allow us to separate the central source from the extended emission.

\subsection{MACS J0329.6-0211}

MACS J0329.6-0211 is the most distant $(z=0.45)$ among the clusters studied here. It is a relaxed system with a global temperature of $\sim 6 \mathrm{keV}$ (Table 5), and it has a bright, lowentropy cool core (Cavagnolo et al. 2009).
Only one pointed observation at $1.4 \mathrm{GHz}$ is available in the VLA archive (Table 2). The observation is in B configuration, and the resulting image is presented in Figure 6(a). A bright point source $(\mathrm{S} 1)$ is coincident with the position of the BCG, as shown by the overlay of the radio emission on the optical HST image (b). The compact source appears surrounded by a heart-shaped diffuse structure, possibly a minihalo, with a radius of $\sim 70 \mathrm{kpc}$ $(+3 \sigma$ isocontour). A slightly smoothed image at $1.4 \mathrm{GHz}$ is shown as grayscale in Figure 6(a) and includes emission below the $3 \sigma$ level. The image suggests that the candidate minihalo extends further out, possibly reaching a distance of $\sim 150 \mathrm{kpc}$ from the center if we include the positive residuals visible to the southeast.

For S1 we measure a flux density of $3.8 \pm 0.2 \mathrm{mJy}$ and a radio power of $(2.8 \pm 0.1) \times 10^{24} \mathrm{~W} \mathrm{~Hz}^{-1}$ on an image obtained by cutting the innermost $15 \mathrm{k} \lambda$ region of the $u-v$ plane to remove the contribution of the surrounding extended emission. The candidate minihalo has $3.8 \pm 0.4 \mathrm{mJy}$ and $P_{1.4 \mathrm{GHz}}=(2.8 \pm 0.3) \times 10^{24} \mathrm{~W} \mathrm{~Hz}^{-1}$.

An overlay of the radio contours on the Chandra image in Figure 6(c) indicates that the diffuse radio emission is centrally located and fills a large portion of the cluster core, as seen in 

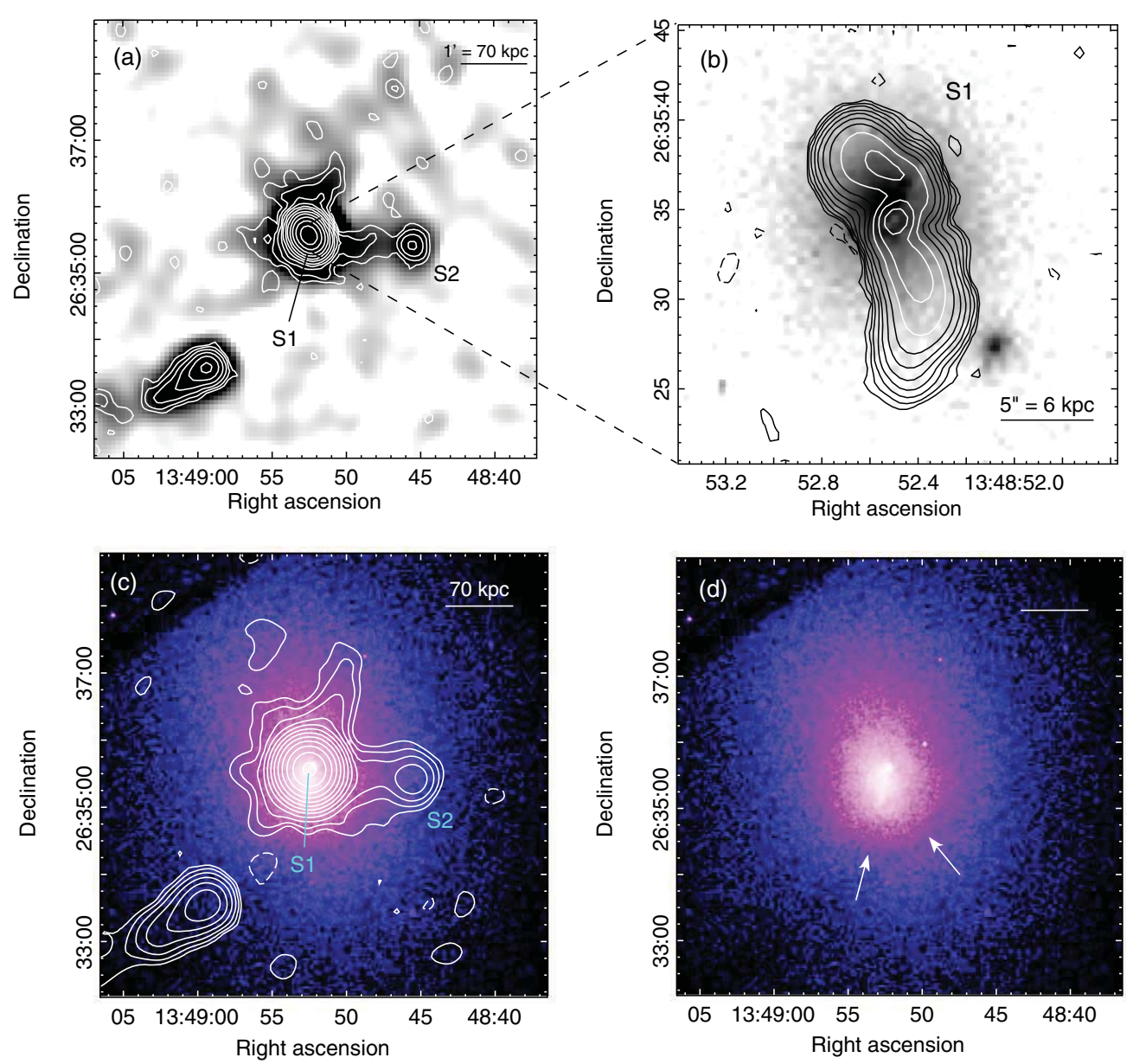

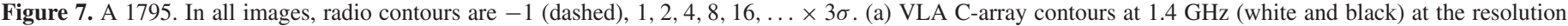

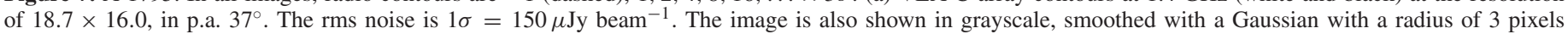

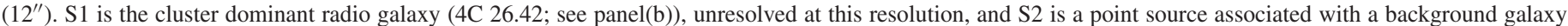

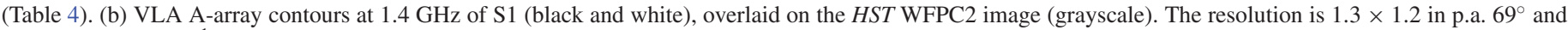

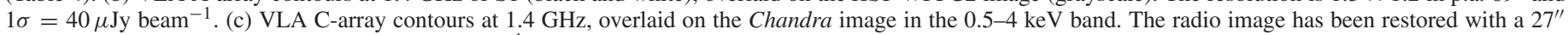
circular beam. The rms noise is $1 \sigma=140 \mu \mathrm{Jy} \mathrm{beam}^{-1}$. (d) Chandra image, the same as in (c). Arrows show the position of a cold front.

(A color version of this figure is available in the online journal.)

other minihalos clusters. The cluster appears very relaxed on large scales. In the core, a surface brightness edge, possibly a cold front, is visible at $\sim 10^{\prime \prime}$ from the center, indicated by the arrow in panel (d).

Deeper radio observations are needed to determine the total extent of the putative minihalo. In addition, as in the case of MACS J0159.8-0849, radio data at higher resolution are necessary to image the radio emission associated with the BCG and investigate its possible connection to the diffuse component.

\subsection{A 1795}

A 1795 is one of the first clusters in which a sloshing cold front has been detected with Chandra (Markevitch et al. 2001). This nearby $(z=0.06)$ cool-core cluster is considered one of the most relaxed clusters in X-rays, showing a very regular Xray morphology (e.g., Ettori et al. 2002). Its cD harbors an FR-I radio galaxy (4C 26.42) with a double Z-shaped morphology and a size of $\sim 12^{\prime \prime}(\sim 14 \mathrm{kpc}$; Ge \& Owen 1993).

Our VLA images at $1.4 \mathrm{GHz}$ are presented in Figure 7. Panel (a) shows the C-array image ( $\sim 19^{\prime \prime}$ resolution), in which 4C 26.42, labeled S1, is unresolved. A second point source (S2) is detected $\sim 1.6$ west of S1. This source coincides with a background galaxy with $z_{\text {phot }}=0.57$ (Table 4). Figure 7(b) shows an arcsecond-resolution image of S1 (in good agreement with Ge \& Owen 1993), overlaid on the optical HST image of the BCG. Figure 7(a) indicates that larger-scale diffuse emission may be present around $\mathrm{S} 1$ and in the region between S1 and S2, suggesting the existence of a central minihalo. The brightness distribution of the candidate minihalo appears unusually filamentary, with two prominent radial extensions pointing northwest and west. The smoothed grayscale image suggests that the minihalo could extend to a radius of $\sim 100 \mathrm{kpc}$, as also shown by an image with a slightly lower resolution overlaid on the Chandra image in Figure 7(c).

To measure the flux density of the candidate minihalo, we subtracted the contribution of S1 and S2 from the total flux density in our low resolution image. For S1, we used the flux density measured on the FIRST ${ }^{11}$ image $(917 \pm 46 \mathrm{mJy}$; not shown here), where the radio galaxy is slightly extended, with its major axis oriented NE-SW, as seen at higher resolution. We note that the FIRST value is between the $890 \pm 45 \mathrm{mJy}$ measured on the A-array image and $960 \pm 48 \mathrm{mJy}$ given by a Gaussian fit to the central point source in the $\mathrm{C}$-array image. For S2, we measured $5.1 \pm 0.3 \mathrm{mJy}$. The resulting flux of the

11 Faint Images of the Radio Sky at Twenty-cm (Becker et al. 1995). 

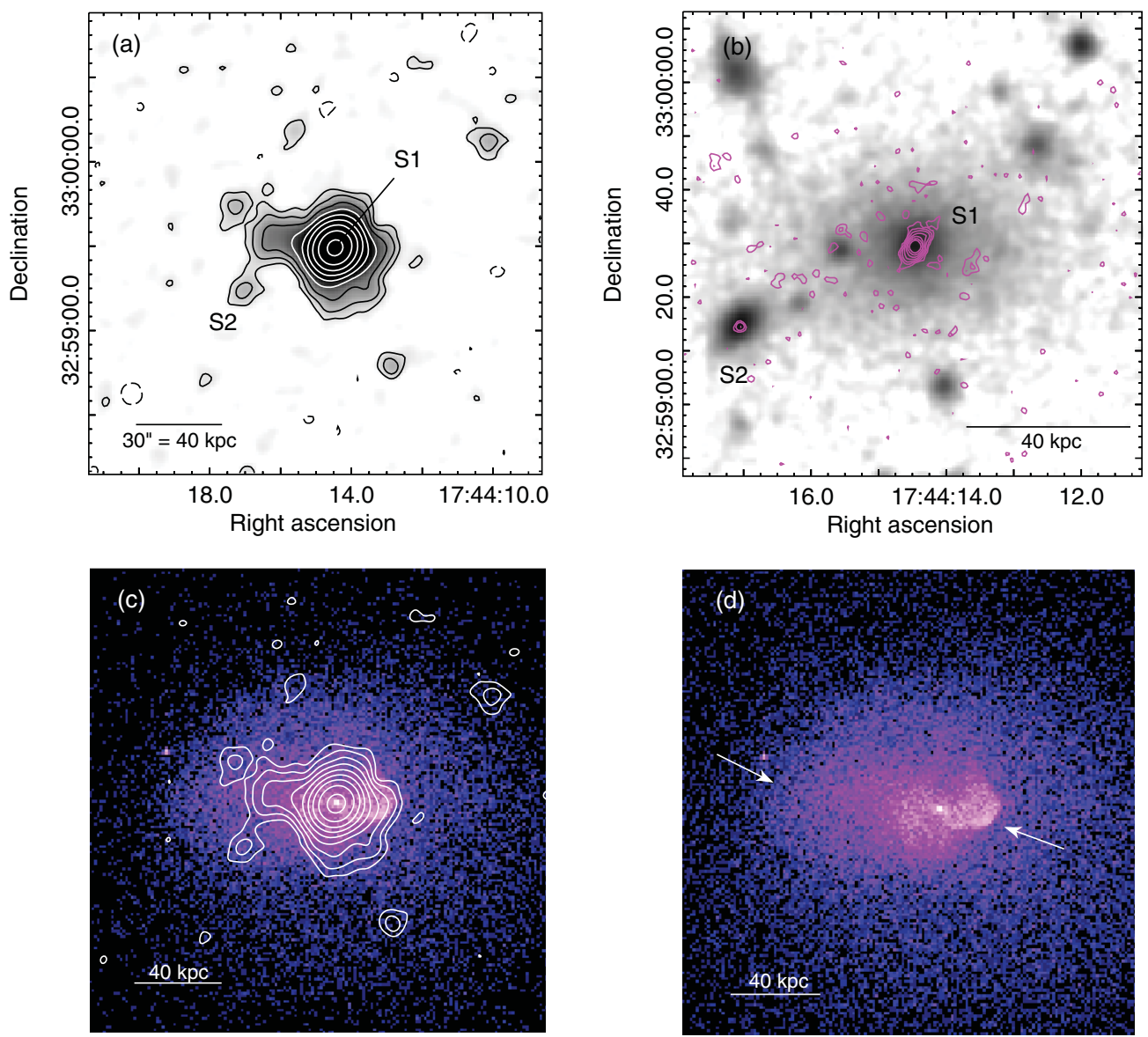

Figure 8. $\mathrm{ZwCl} 1742.1+3306$. (a) VLA DnA-configuration contours at $1.4 \mathrm{GHz}$ (black and white). The restoring beam is $10.7 \times 9.7$ in p.a. -40 . The rms noise is $1 \sigma=70 \mu \mathrm{Jy} \mathrm{beam}^{-1}$, and contours are -1 (dashed), $1,2,4,8,16, \ldots \times 3 \sigma$. The image is also shown in grayscale, smoothed with a Gaussian with a radius of $3^{\prime \prime}$. The unresolved source S1 is associated with the BCG, and S2 is coincident with a nearby galaxy without redshift (see panel (b) and Table 4). (b) VLA-DnA full-resolution contours at $1.3 \mathrm{GHz}$ (magenta), overlaid on the optical POSS -2 image (grayscale). The restoring beam is 1". $50 \times 11^{\prime \prime} 49$ in p.a., 48 and $1 \sigma=65 \mu \mathrm{Jy}$ beam ${ }^{-1}$. Contours scale as in (a). No levels at $-3 \sigma$ are present in the portion of the image shown. (c) $1.3 \mathrm{GHz}$ contours (the same as in (a)) overlaid on the Chandra image in the 0.5-4 keV band. The image has the same physical size as panel (a). (d) Chandra image, the same as in (c). Arrows indicate the position of two cold fronts.

(A color version of this figure is available in the online journal.)

minihalo candidate is $85 \pm 5 \mathrm{mJy}$, corresponding to a radio power at $1.4 \mathrm{GHz}$ of $(7.9 \pm 0.5) \times 10^{23} \mathrm{~W} \mathrm{~Hz}^{-1}$.

The prominent cold front discovered by Markevitch et al. (2001) in the Chandra image is located $\sim 70^{\prime \prime}$ south of the center (arrows in Figure 7(d)). The radio/X-ray comparison in panel (c) suggests that the cold front may be confining the southern emission of the minihalo.

Deeper radio observations are necessary to confirm the presence of a central minihalo in this cluster and to determine its total size and possible association with the cold front.

\section{4. $\mathrm{ZwCl} 1742.1+3306$}

$\mathrm{ZwCl} 1742.1+3306$ is a nearby $(z=0.076)$ cool-core cluster with a global temperature of $\sim 5 \mathrm{keV}$ (Table 5). A recent Chandra detection of two well-defined cold fronts at $\sim 20 \mathrm{kpc}$ west and $\sim 80 \mathrm{kpc}$ east of the BCG indicates that the cool core is sloshing (Ettori et al. 2013; see also Figure 8(d)). Two possible small cavities are located a few tens of kpc north and south of the BCG (Ettori et al. 2013).

We imaged the cluster center at $1.4 \mathrm{GHz}$ using the DnAconfiguration observation in Table 2. The angular resolution of the full array is $1^{\prime \prime}$. We also obtained images with slightly lower angular resolutions by weighting down long baseline data points. Our lowest-resolution image $\left(\sim 10^{\prime \prime}\right)$ is shown in
Figure 8(a). Extended emission is present at the cluster center around the central radio galaxy S1. At arcsecond resolution, the source $\mathrm{S} 1$ is dominated by a bright compact component (Figure 8(b)), whose peak is coincident with the central active galactic nucleus (AGN) and is detected as a point source in X-rays (Ettori et al. 2013, see also Figure 8(d)). Two weak, extended components are located NW and SW of the center, pointing to, but not reaching, the two X-ray cavities (Figure 8(d)). A point source (S2) is detected at the position of a galaxy without redshift information.

Gregory \& Condon (1991) report a flux density of $74 \mathrm{mJy}$ at $4.9 \mathrm{GHz}$ for the central radio galaxy. We measure $69 \mathrm{mJy}$ at $1.4 \mathrm{GHz}$, which implies a flat (possibly inverted) spectrum $(\alpha=$ -0.1 ). This is consistent with the core-dominated morphology of S1 in Figure 8(b). After the subtraction of the flux densities of S1 and S2 (Table 4) from the total emission in Figure 8(a), we estimate a residual flux density of $13.8 \pm 0.8 \mathrm{mJy}$ in diffuse emission, which corresponds to a luminosity of $(2.0 \pm 0.1) \times$ $10^{23} \mathrm{~W} \mathrm{~Hz}^{-1}$.

A bright, unresolved source is detected at the cluster center by the $\operatorname{VLSSR}^{12}$ at $74 \mathrm{MHz}$ and WENNS ${ }^{13}$ at $325 \mathrm{MHz}$, with flux

\footnotetext{
12 VLA Low-Frequency Sky Survey Redux (Lane et al. 2012).

13 The Westerbork Northern Sky Survey (Rengelink et al. 1997).
} 
densities of $2.4 \mathrm{Jy}^{14}$ and $332 \mathrm{mJy}$, respectively, giving $\alpha=1.3$ for the whole emission (S1+diffuse component). Although higher resolution images at these frequencies are needed to separate the large-scale emission from the central source S1, this spectral index suggests that the extended emission seen in Figure 8(b) has a steep spectrum.

Figure 8(c) shows the Chandra image with an overlay of the $1.4 \mathrm{GHz}$ contours. Within the central $r \sim 30 \mathrm{kpc}$, the diffuse radio emission is relatively round and covers the brightest $\mathrm{X}$-ray emission in the core. On larger scales, the radio emission extends toward the east, out to a radius of $\sim 40 \mathrm{kpc}$, following an elongation of the X-ray emission toward the eastern cold front (Figure 8(d)). Overall, the radio emission appears contained within the sloshing region defined by the cold fronts, although at this brightness level it does not reach the eastern front.

As we will discuss in Section 6.5, the nature of the extended emission detected in this cluster is unclear. Further radio observations are necessary to investigate the relationship between the central radio galaxy and the larger-scale component.

\subsection{MACS J1931.8-2634}

MACS J1931.8-2634 $(z=0.35)$ is a relaxed and massive cluster that harbors one of the most X-ray luminous cool cores known, with a bolometric luminosity of $\sim 1 \times 10^{45} \mathrm{erg} \mathrm{s}^{-1}$ and a cooling time $<1 \mathrm{Gyr}$ in its innermost $r \sim 50 \mathrm{kpc}$ region (Ehlert et al. 2011).

A combined X-ray, optical, and radio study of this system has been presented by Ehlert et al. (2011), showing that the cluster core has been significantly disturbed by both AGN activity and sloshing of the low-entropy gas along a roughly N-S direction, as indicated by a prominent spiral structure in the temperature map. Two possible X-ray cavities are located to the east and west of the central powerful AGN, which is a bright X-ray source (Ehlert et al. 2011; see also Figure 9(f)). Using the VLA, Ehlert et al. (2011) imaged the cluster core at $1.4 \mathrm{GHz}$ and found a central compact source, coincident with the BCG and extended, amorphous emission on a larger scale.

We reprocessed these VLA observations (Table 2). Our images are shown in Figure 9. As previously found, the emission in the cluster core is composed by a bright, unresolved source (S1), wholly enclosed in a much fainter, diffuse structure $\sim 100 \mathrm{kpc}$ long in the $\mathrm{E}-\mathrm{W}$ direction and $\sim 60 \mathrm{kpc}$ in the perpendicular direction (Figure 9(a)). No distinct jets or lobes are seen in the extended emission. A second compact component (S2) is detected $\sim 3^{\prime \prime}$ from S1. To better separate S1 and S2 from the surrounding extended emission, we produced an image from the A-configuration data using only the baselines longer than $20 \mathrm{k} \lambda$. In Figure 9(b) we show the resulting image overlaid on the optical HST image of the BCG. The peak of S1 is coincident with the central AGN source. S2 has no clear association with point sources on the $H S T$ image, but is cospatial with a bright $\mathrm{H} \alpha$ and a blue light filament to the northwest of the BCG, which also coincides with a bright, cool, and metal-rich ridge of X-ray emission (Ehlert et al. 2011).

The $1.4 \mathrm{GHz}$ image from the $\mathrm{B}$ configuration is presented in Figure 9(c). The source is more extended than imaged at higher resolution and reaches a size of $R_{\max } \sim 100 \mathrm{kpc}$ and $R_{\text {min }} \sim 90 \mathrm{kpc}$, which is almost twice the size seen in the Aconfiguration image in panel (a). The source is also detected with the VLA at $327 \mathrm{MHz}$ on a similar spatial scale (image not shown here), but it is only marginally resolved due to the relatively

\footnotetext{
$\overline{14}$ This value is in the Scaife \& Heald (2012) flux density scale.
}

low angular resolution (Table 2). Inspection of a $150 \mathrm{MHz}$ image from the $\mathrm{TGSS}^{15}$ reveals that the source is even more extended. The $150 \mathrm{MHz}$ image is reported in panel (d) with the $1.4 \mathrm{GHz}$ contours overlaid for comparison. The emission seen at low frequency appears slightly tilted with respect to the E-W emission at $1.4 \mathrm{GHz}$ and reaches a large size of $R_{\max } \sim 300 \mathrm{kpc}$ along its NE-SW major axis. The marginally extended source to the south is resolved at $1.4 \mathrm{GHz}$ into a narrow-angle tail (NAT) associated with a bright galaxy $\sim 40^{\prime \prime}$ (projected) from the BCG (see also Ehlert et al. 2011).

The flux densities at $1.4 \mathrm{GHz}$ of S1 and S2 are $11.6 \pm 0.6 \mathrm{mJy}$ and $2.5 \pm 0.1 \mathrm{mJy}$, respectively, as measured on the image in Figure 9(b). A total of $62 \pm 3 \mathrm{mJy}$ is found in the lowresolution, B-array image in Figure 9(c). After subtraction of the contributions of $\mathrm{S} 1$ and S2, $48 \pm 3 \mathrm{mJy}$ can be attributed to the outer diffuse emission, which is therefore the dominant component in the system with $\sim 80 \%$ of the total flux density. Its radio power is $(2.0 \pm 0.1) \times 10^{25} \mathrm{~W} \mathrm{~Hz}^{-1}$. Due to the lower angular resolution of the $327 \mathrm{MHz}$ and $150 \mathrm{MHz}$ images, we are not able to separate the individual components and can only obtain a flux density for the whole system. We measure $669 \pm 33 \mathrm{mJy}$ at $327 \mathrm{MHz}$ and $6.15 \pm 1.50 \mathrm{Jy}$ at $150 \mathrm{MHz}$. The inferred spectral index between $150 \mathrm{MHz}$ and $1.4 \mathrm{GHz}$ is very steep, $\alpha=2.1 \pm 0.1$.

In Figure 9(e), we overlay the $1.4 \mathrm{GHz}$ contours on the Chandra image. The diffuse emission covers the entire core region and is far more extended than the region occupied by the possible X-ray cavities, whose association with the radio emission is therefore unclear. On the other hand, the axis connecting the cavities and major axis of the radio emission are oriented in a similar direction, suggesting a connection between the radio and X-ray features. Furthermore, as noticed by Ehlert et al. (2011), the outer boundaries of the putative cavities are not well defined. It is therefore possible that the cavities are larger or that additional, older cavities are present further away from the center.

Due to the ultrasteep radio spectrum of the radio emission, its highly elongated and much wider structure at low frequency, and its unclear link with the X-ray cavities, we consider the classification of this source as a minihalo to be very uncertain, as discussed in more detail in Section 6.5.

\section{DISCUSSION}

Our current knowledge of radio minihalos in cluster cores has been based on only 12 reported objects (Table 5), which is insufficient to investigate their origin and answer the question of why they are so rare. It is therefore crucial to look for more minihalos in a systematic way. We have selected a large sample of X-ray luminous clusters with available high-quality radio data in order to search for new possible minihalos. Our goal is to investigate the radio properties of these clusters in relation to their global and core properties (S. Giacintucci et al., in preparation). In this article, we have presented nine clusters from the sample, and we found clear indications of a centrally located diffuse radio emission around the radio active BCG in their VLA images. We give a brief summary of our findings below.

1. Based on the available radio information at both high and low angular resolutions, we have found central diffuse

\footnotetext{
15 TIFR GMRT Sky Survey (S. K. Sirothia et al., in preparation),
} http://tgss.ncra.tifr.res.in/150MHz/tgss.html. 

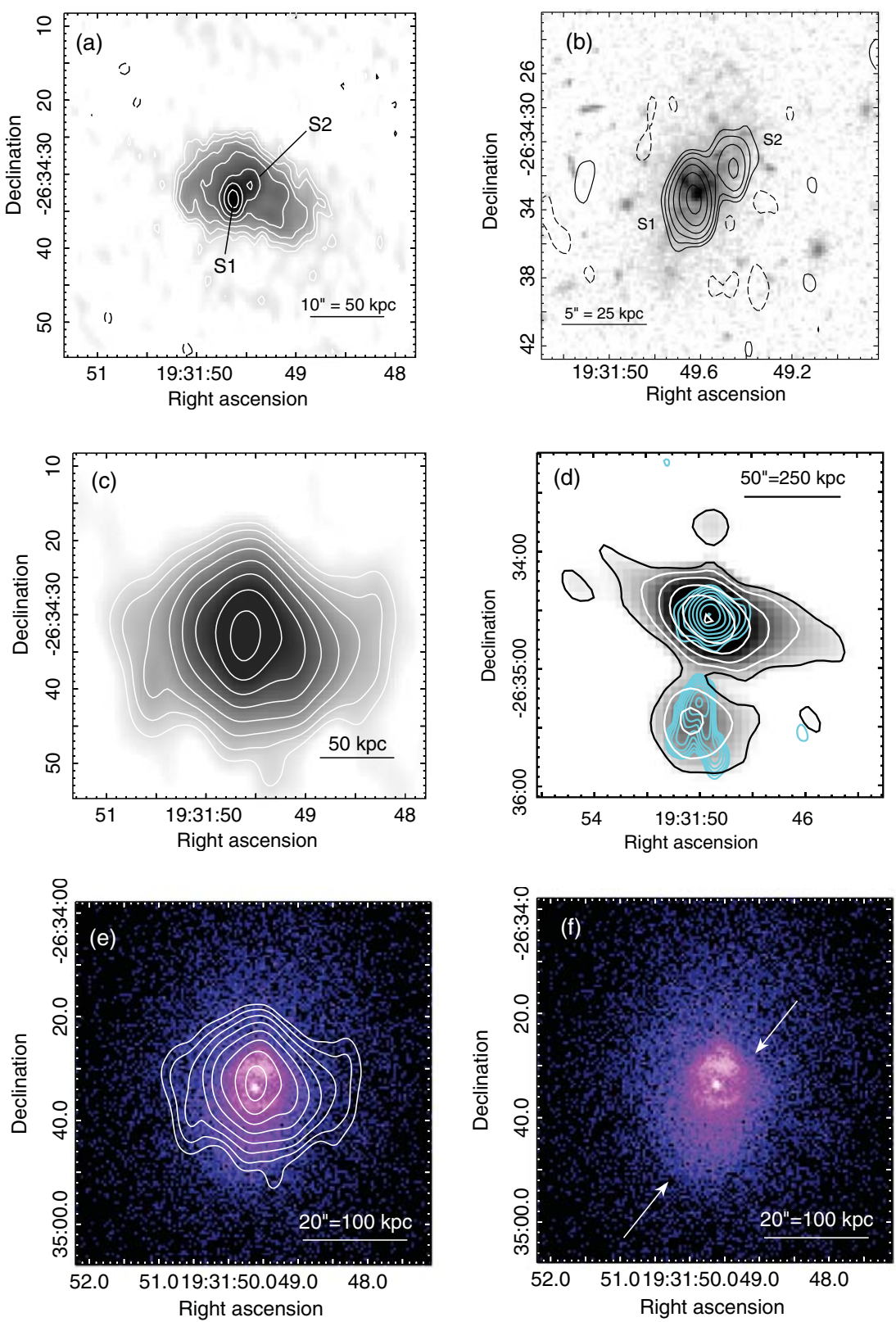

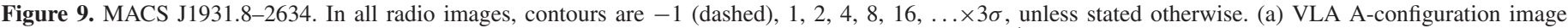

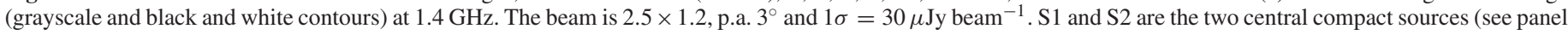

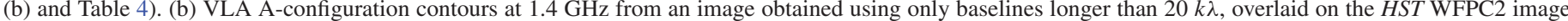

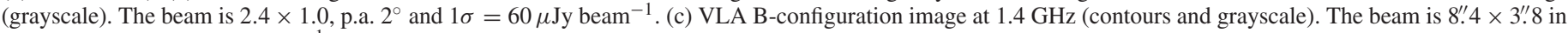

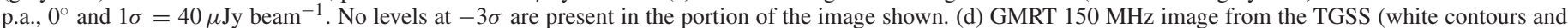

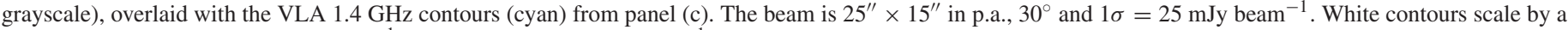

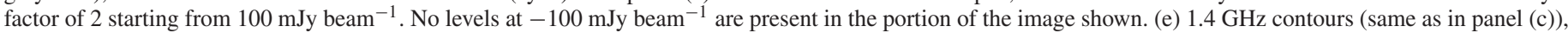

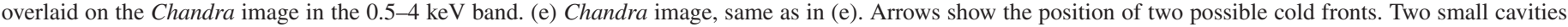
are visible to the east and west of the central AGN (see Ehlert et al. 2011).

(A color version of this figure is available in the online journal.)

emission in A 478, RX J1532.9+3021, ZwCl 3146 and A 2204 that is consistent with a minihalo. In these clusters, we were able to image the central radio galaxy on a scale $r \lesssim 10 \mathrm{kpc}$, that is considerably smaller than the extent of the surrounding minihalo $\left(R_{\mathrm{MH}}>50 \mathrm{kpc}\right)$, and found no connection (for instance, in the form of jets or lobes) between the radio galaxy and the outer extended emission.

2. In MACS J0159.8-0849 and MACS J0329.6-0214, the two most distant systems among the clusters presented here, the existing data do not allow us to map the emission from the central radio galaxy on scales of $r \lesssim 100 \mathrm{kpc}$.
Thus we cannot rule out a direct connection between radio galaxy and larger-scale diffuse emission, which we therefore classify as candidate minihalos.

3. We found a candidate minihalo in A 1795, where hints of a very faint, diffuse source of a much larger linear size than the central radio galaxy $4 \mathrm{C} 26.42$ can be seen in the $1.4 \mathrm{GHz}$ image.

4. We detected extended emission enshrouding the dominant radio galaxy in ZwCl 1742.1+3306 and MACS J1931.82634. However, the interpretation of this emission as a minihalo is uncertain and requires further radio observations. 


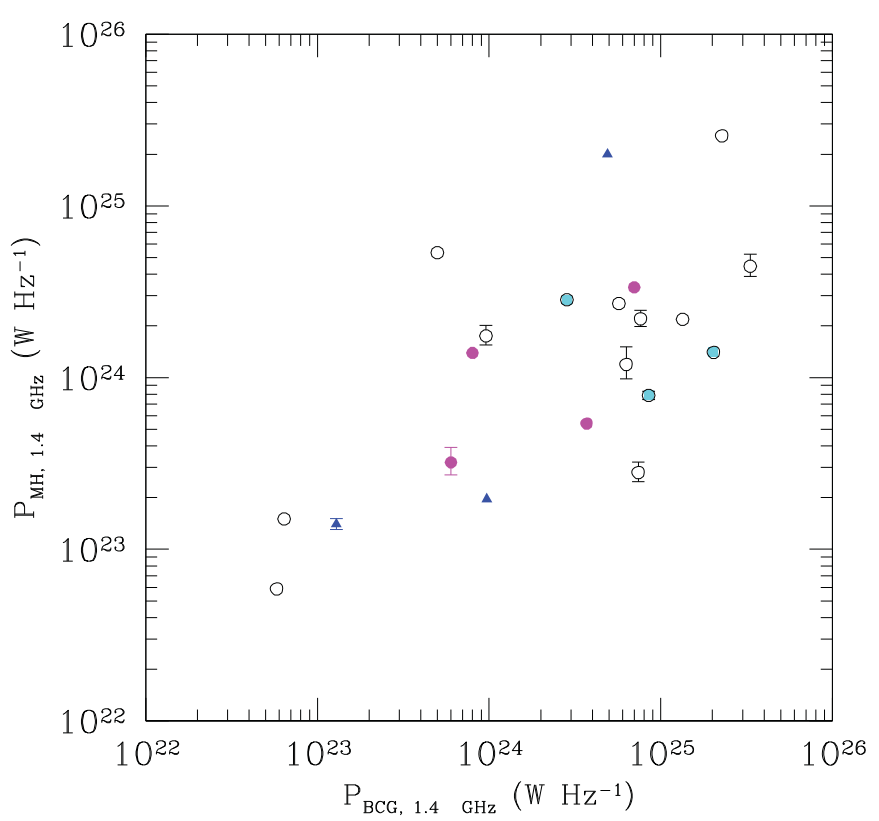

Figure 10. $P_{\mathrm{MH}, 1.4 \mathrm{GHz}}-P_{\mathrm{BCG}}, 1.4 \mathrm{GHz}$ Diagram for the clusters with previously known minihalos (empty black circle), new minihalo detections (magenta circles), minihalo candidates (cyan circles), and central extended sources whose classifications as minihalos are uncertain (blue triangles). Only error bars corresponding to an uncertainty of $>10 \%$ on the radio power are plotted.

(A color version of this figure is available in the online journal.)

These sources will be discussed in more detail in Section 6.5 .

The new minihalos and candidates and the previously known minihalos reported in Table 5 are all well detected. Even for the least significant detection-the candidate minihalo in MACS J0329.6-0214-the brightest part of the minihalo outside of the central point source is imaged at $\sim 7 \sigma$ with respect to the image noise (per beam), and the best cases are imaged at more than $50 \sigma$.

\subsection{Radio Properties of the BCG in Minihalo Clusters}

By comparing the radio power of the BCGs and the radio luminosity of the surrounding minihalos in six clusters, Govoni et al. (2009) noticed that stronger minihalos tend to occur in clusters with more powerful central radio galaxies, suggesting that the minihalo emission could be partially related to the activity of the central AGN. For our larger sample of minihalos and candidates, we find a possible weak trend, although with a very large scatter (Figure 10). A similar trend is also visible in the flux-flux plane, indicating that the possible relation between minihalo and BCG luminosities may be intrinsic. We find a Spearman rank correlation coefficient of $r_{s} \sim 0.5$ in both planes with a probability of no correlation of a few percent.

This result suggests that the central AGN activity is not directly powering the minihalo emission, although it is one of the plausible sources of the seed relativistic electrons for the reacceleration models (Cassano et al. 2008). As argued by Govoni et al. (2009), a tight correlation between the minihalo and BCG radio properties is not expected, as the radio galaxy likely undergoes multiple cycles of activity within the lifetime of the minihalo, which is supported by the evidence for recurrent radio outbursts for a number of cluster and group dominant galaxies (e.g., Clarke et al. 2009; David et al. 2009; Giacintucci et al. 2011b; Randall et al. 2011; Giacintucci et al. 2012, Venturi et al. 2013).

\subsection{Comparison between Radio Minihalos and Global Properties of the Cluster Hosts}

Cassano et al. (2008) found that clusters with higher X-ray luminosity tend to possess more powerful minihalos. However, this result was based on the only six minihalos that were known at the time of their study. A more recent investigation was carried out by Kale et al. (2013) for a larger number of minihalo clusters (11, 5 of which were from the GRHS cluster sample). Despite the large scatter, they found indication of a possible radio/X-ray luminosity correlation, suggesting that an intrinsic relation between the thermal and nonthermal cluster properties may exist.

We can explore this possibility further using our larger sample of 21 minihalo clusters. In Figure 11(a), we plot the minihalo radio power at $1.4 \mathrm{GHz}$ versus the cool core-excised cluster temperature (Table 5), which can be used as a proxy for the cluster total mass and is strongly correlated with the X-ray luminosity. Our comparison does not indicate a clear scaling between radio power and cluster temperature, regardless of whether we include candidates or exclude them. We indeed find $r_{s} \sim 0.3$ and $P_{\text {no corr }} \sim 20 \%\left(r_{s} \sim 0.1\right.$ and $P_{\text {no corr }} \sim 70 \%$ if we consider only clear minihalo detections).

However, the plot provides us an important piece of information on the kind of clusters that possess a minihalo at their center: they all tend to have high global temperatures, with the majority of minihalos found in $T>5 \mathrm{keV}$ systems. While proper statistical analysis should include nondetections (S. Giacintucci et al., in preparation), this suggests that the hosts of our detected minihalos are massive clusters.

An alternative way to estimate cluster masses is offered by the SZ effect (e.g., Carlstrom et al. 2002). Of the 21 minihalo clusters considered here, 14 are in the all-sky cluster catalog of validated clusters from the first 15.5 months of Planck observations (Planck Collaboration et al. 2013). In Table 5, we report their total masses within $R_{500}$, inferred from the Planck observations. Figure 11(b) shows the distribution of our subsample of minihalo clusters that have Planck data in the $P_{\mathrm{MH}, 1.4 \mathrm{GHz}}-M_{500}$ plane. No obvious correlation is visible between the radio luminosity and cluster mass-in this case we find $r_{s} \sim 0.3$ and $P_{\text {nocorr }} \sim 10 \%$-which is in agreement with the lack of a clear correlation with the global temperature in panel (a). We note that this is in contrast with the giant radio halos found in cluster mergers, whose radio luminosity correlates with the cluster mass (Cassano et al. 2013 and references therein). Again, we find evidence that minihalos are hosted by massive clusters, as all minihalos are in $M_{500} \gtrsim 5 \times 10^{14} M_{\odot}$, except for the slightly less massive system 2 A $0335+096\left(M_{500}=2 \times 10^{14} M_{\odot}\right)$.

In a subsequent article, we will investigate possible correlations with the thermodynamical properties of the cool cores rather than the global cluster properties (S. Giacintucci et al., in preparation).

\subsection{Spectral Properties of Minihalos}

Until now, our knowledge of the radio spectra of minihalos has been limited to only two sources, Perseus and Ophiuchus, whose integrated spectra are based on flux density measurements at three frequencies. Figure 12 shows these spectra from Sijbring (1993) and Murgia et al. (2010). In black, we show the spectrum of a new minihalo detected in RX J1532.9+3021, based on flux densities at four frequencies; this is reported in Section 4.2 (note that the spectrum has been multiplied by 100 for display 

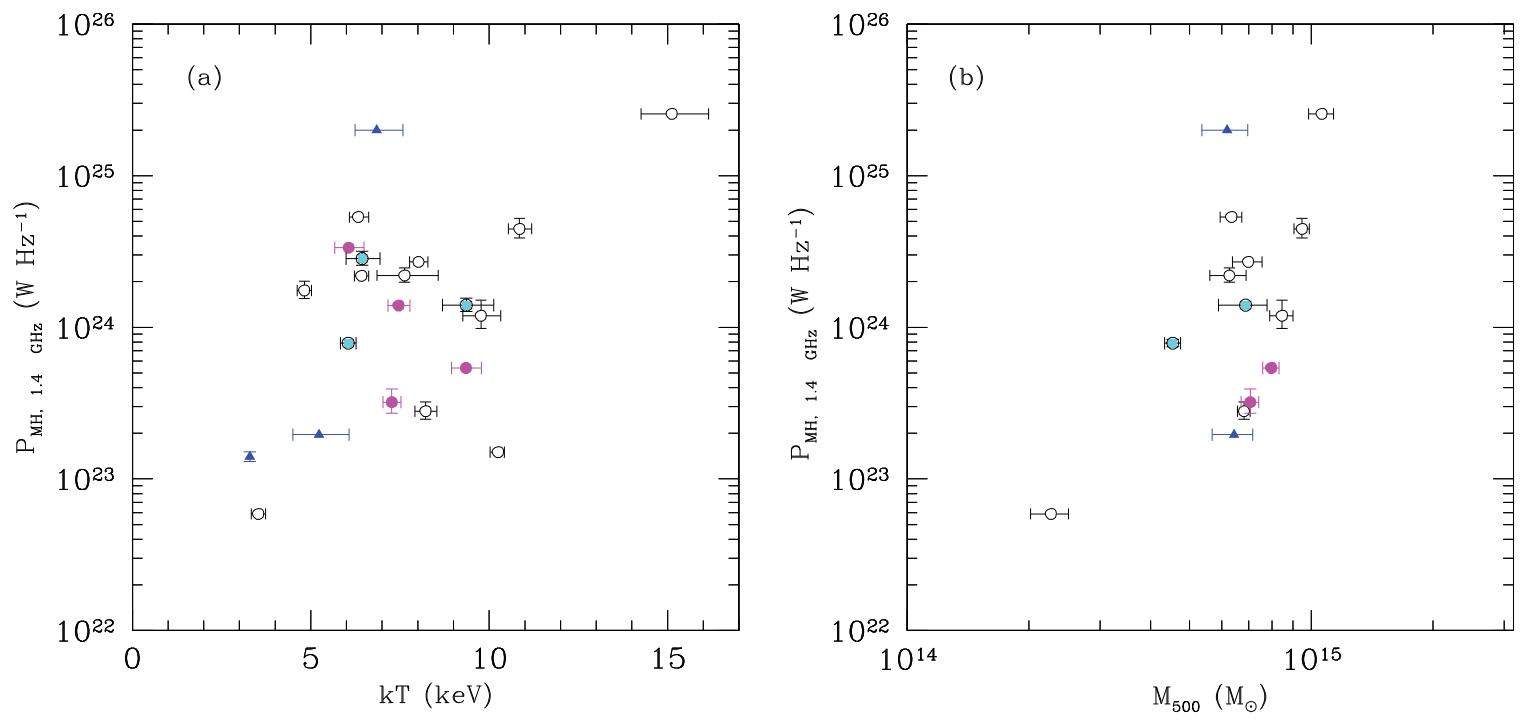

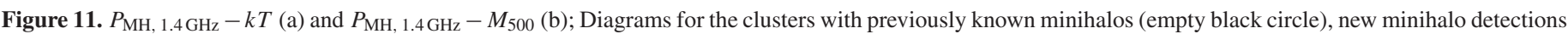

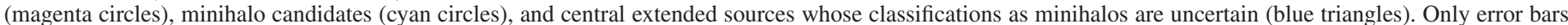
corresponding to an uncertainty of $>10 \%$ on the radio power are plotted.

(A color version of this figure is available in the online journal.)

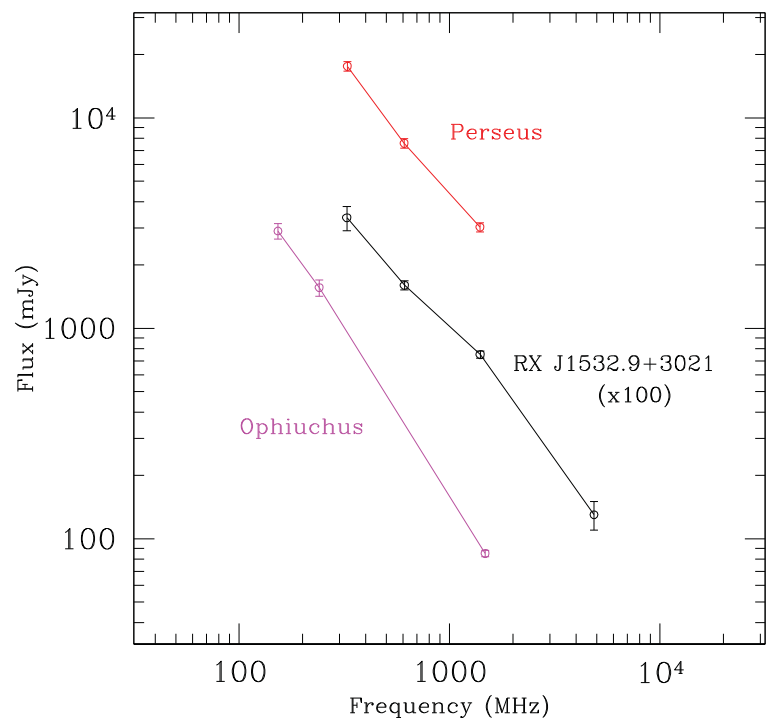

Figure 12. Integrated radio spectra of the minihalos in RX J1532.9+3021 (black), Perseus (red, from Sijbring 1993), and Ophiuchus (magenta, from Murgia et al. 2010). The total spectral index is $\alpha=1.20 \pm 0.03$ for RX J1532.9+3021, $\alpha=1.21 \pm 0.05$ for Perseus, and $\alpha=1.56 \pm 0.04$ for Ophiuchus. Note that the spectrum of RX J1532.9+3021 has been multiplied by 100.

(A color version of this figure is available in the online journal.)

purposes). The three spectra appear very similar in shape, at least in the range of frequencies currently explored. They all seem to be well described by a power law with a steep spectral index $\alpha=1.21 \pm 0.05$ and $\alpha=1.20 \pm 0.07$ for Perseus and RX J1532.9+3021, and a slightly steeper slope for Ophiuchus, $\alpha=1.56 \pm 0.04$. For Ophiuchus, a steepening at the high frequency may be present, although the spectral indices below and above the data point at $610 \mathrm{MHz}$ are consistent within the errors (Murgia et al. 2010). A steepening may be also present in the spectrum of RX J1532.9+3021, where the spectral index changes from $\alpha=1.02 \pm 0.10$ between $325 \mathrm{MHz}$ and $1.4 \mathrm{GHz}$ to $\alpha=1.41 \pm 0.13$ above $1.4 \mathrm{GHz}$.
The existing spectral information is not sufficient to discriminate between the competing models for the minihalo formation, i.e., a power-law spectrum over the entire radio frequency range expected in pure secondary models vs. a high-frequency break predicted by turbulent reacceleration models. More data points and a frequency range wider than that shown in Figure 12 are necessary to accurately determine the shape of the minihalo spectra and to confirm the high-frequency steepening in Ophiuchus and RX J1532.9+3021.

\subsection{Is Particle Acceleration Needed in Small-size Minihalos?}

As for the megaparsec-sized, giant radio halos found in many massive, merging clusters, the few hundred kiloparsec scale of large minihalos (such as Perseus and Ophiuchus) requires that the relativistic electrons are continuously injected and/ or reaccelerated in situ throughout the large emitting volume. Indeed, in the strong magnetic fields expected in the cool cores, the radio-emitting electrons cannot diffuse from the central radio galaxy out to the minihalo radius within their radiative lifetime, which is almost two orders of magnitude shorter than their diffusion timescale (e.g., Jaffe 1977).

The question we pose here is whether diffusion can still account for smaller minihalos, such as the $R_{\mathrm{MH}} \sim 50 \mathrm{kpc}$ emission in A 2204. The typical diffusion velocity of relativistic electrons in clusters is expected to be approximately the Alfvén velocity (Jaffe 1977) $v_{A}=B /(4 \pi \rho)^{(1 / 2)}$, where $B$ is the cluster magnetic field and $\rho$ is the density of the ICM. Although our knowledge of the magnetic field in clusters is still poor, studies of the rotation measure of radio galaxies in or behind clusters indicate that the magnetic field intensity in cool cores can be as high as $5-10 \mu \mathrm{G}$ or even stronger (e.g., Taylor et al. 2002; Clarke 2004). In these strongly magnetized, high-density $\left(n_{\mathrm{e}} \sim 10^{-2} \mathrm{~cm}^{-3}\right)$ cluster regions, $v_{A} \sim 100 \mathrm{~km} \mathrm{~s}^{-1}$. At this speed, the time required for the electrons to reach a radius of $\sim 50 \mathrm{kpc}$ from the center is $\sim 5 \times 10^{8} \mathrm{yr}$, which needs to be compared to their synchrotron radiative cooling time of $\sim 10^{7} \mathrm{yr}$.

Thus, even the small minihalos like the one in A 2204 cannot be produced by electrons leaking from the central radio galaxy and diffusing within the cluster core volume. However, other 
physical mechanisms may be able to transport the particles from the radio galaxy volume to larger radii faster. For instance, largescale sloshing motions can potentially advect the electrons along with the gas and redistribute them throughout the sloshing region (e.g., ZuHone et al. 2013, see also discussions in Gastaldello et al. 2013 and Venturi et al. 2013). If we assume a sloshing velocity of half the sound speed for a rough estimate, the electrons could be carried out to a distance of $\sim 30-40 \mathrm{kpc}$ from the center within $10^{7} \mathrm{yr}$, suggesting that this kind of transport mechanism may indeed play a role in the less-extended minihalos.

\subsection{ZwCl 1742 and MACS J1931: A Radio Minihalo, Fading Radio Lobes, or Something in Between?}

As shown in Sections 5.4 and 6.5, the radio information available for the extended radio sources in the cores of ZwCl 1742.1+3306 and MACS J1931.8-2634 is not sufficient to unambiguously classify the central emission as a minihalo; further observations are needed to understand their nature.

In MACS J1931.8-2634, the diffuse emission is rather elongated and seems confined, despite its amorphous appearance. Part of the emission is found to be cospatial with two possible small, inner X-ray cavities aligned in the same E-W direction as the larger-scale radio emission. A further complication is that the emission at $150 \mathrm{MHz}$ is significantly more extended than those at higher frequencies; the size of the source reaches $\sim 600 \mathrm{kpc}$ in diameter, compared to $\sim 200 \mathrm{kpc}$ at $1.4 \mathrm{GHz}$, and its major axis is slightly tilted with respect to the major axis of the high-frequency emission. Furthermore, the total spectrum is found to have an ultrasteep slope $(\alpha \sim 2)$. As seen in Section 6.3, minihalos tend to have a spectrum with $\alpha \sim$ $1.2-1.5$, at least in those few cases where spectral information is available. Ultrasteep spectra are instead common for dying or restarted radio galaxies, whose aged emission is no longer fed by the nucleus, which is rapidly fading (e.g., Saikia \& Jamrozy 2009; Murgia et al. 2011; Giacintucci et al. 2012, Venturi et al. 2013 and references therein). Thus, the ultrasteep spectrum argues against a minihalo for an earlier radio outburst of the BCG, which is now undergoing a new phase of activity. This is similar to what is seen, for instance, at the center of the NGC 1407 group (Giacintucci et al. 2012). The different orientation of the low- and high-frequency extended structures may also indicate two different (past) radio outbursts that occurred at different jet angles.

On the other hand, the X-ray data indicate that the core of MACS J1931.8-2634 is sloshing in the N-S direction. For a similar sloshing configuration, the simulated minihalo maps by ZuHone et al. (2013) strikingly resemble the radio emission seen at the center of MACS J1931.8-2634 (see their Figure 11): the diffuse emission tends to be distributed in two bright regions that are symmetrical with respect to the cluster center and are aligned in a direction roughly perpendicular to the axis of sloshing.

The source in $\mathrm{ZwCl} 1742.1+3306$ could also be classified as a restarted radio galaxy. However, no detailed spectral information is available to rule out the minihalo interpretation. We notice that the source is only $\sim 40 \mathrm{kpc}$ in radius and, as discussed in Section 6.4, electron reacceleration may not be needed to explain the presence of diffuse emission on such a scale. If relativistic electrons have been injected by the central AGN during a former cycle of activity and accumulated in two radio bubbles, it is possible that ongoing sloshing motions have disrupted such bubbles and carried the aged but still radio-

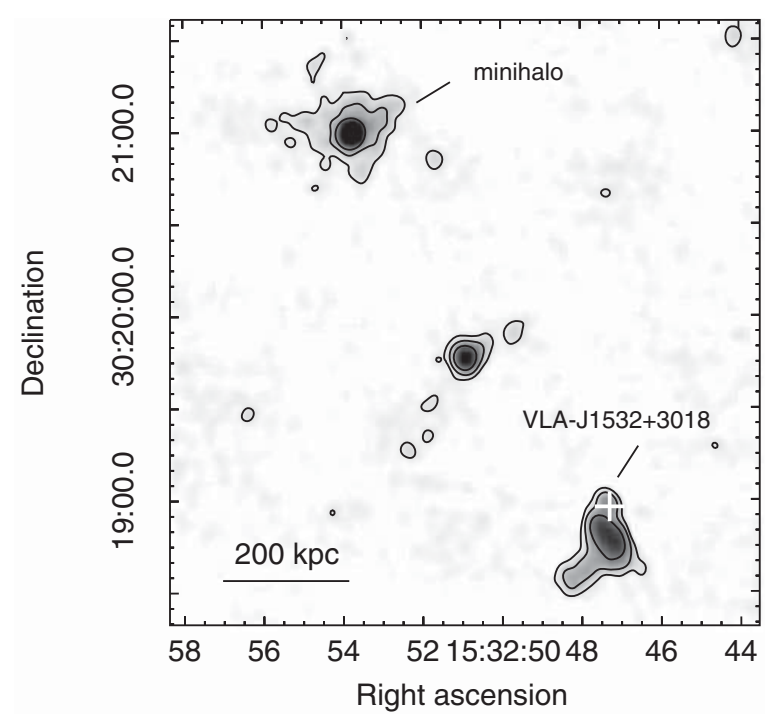

Figure 13. VLA B-array image at $1.4 \mathrm{GHz}$ of the field of RX J1532.9+3021, hosting a radio minihalo at its center (see also Figure 2). A radio source with complex morphology (VLA-J1532+3018) is located $\sim 2$ '.6 southeast of the RX J1532.9+3021 center. The source is associated with the optically selected cluster GMBCG J233.19725+30.31626, whose center is marked as a white cross. The restoring beam is $4^{\prime \prime} .2 \times 3^{\prime \prime} .7$ in p.a. $-11^{\circ}$, and contours are 0.045 , 0.18 and $0.72 \mathrm{mJy}^{\text {beam }}{ }^{-1}$.

emitting electrons throughout the sloshing region. Thus, this object may be an intermediate case between a "pure" minihalo emission arising from reaccelerated electrons and an active extended radio galaxy that is perturbed, but not disrupted, by large-scale sloshing motions, as possibly seen in the wide-angle tail at the center of A 2029 (Clarke et al. 2004; Paterno-Mahler et al. 2013) and in the radio galaxy at the center of A 3560 (Venturi et al. 2013).

For both clusters, further multifrequency radio observations are needed to discriminate between these interpretations by deriving the spectrum of the various contributors to the total emission and by mapping the distribution of the spectral index.

\section{SUMMARY AND CONCLUSIONS}

We have undertaken a systematic search for new radio minihalos in a large sample of X-ray luminous clusters with available high-quality VLA and/or GMRT radio data. In this article, we present new minihalos and minihalo candidates found in nine clusters in the course of our reanalysis of the archival VLA observations. In particular, we found new minihalos in the cores of A 478, RX J1532.9+3021, ZwCl 3146, and A 2204, as well as a candidate minihalo, with an unusually filamentary morphology in A 1795. Diffuse radio emission is also found in the cores of MACS J0159.8-0849 and MACS J0329.6-0214. The existing data for the latter two clusters do not allow us to rule out the possibility of this emission being part of their central radio galaxies; therefore we list them among the minihalo candidates. Other new minihalo candidates include ZwCl 1742.1+3306 and MACS J1931.8-2634; further radio observations are required to determine if these are restarted radio galaxies or minihalos.

The radio luminosities of our minihalos and candidates range between $10^{23} \mathrm{~W} \mathrm{~Hz}^{-1}$ and $10^{25} \mathrm{~W} \mathrm{~Hz}^{-1}$ at $1.4 \mathrm{GHz}$, as commonly found for this type of radio sources. Their sizes $\left(R_{\mathrm{MH}}=40-160 \mathrm{kpc}\right)$ are somewhat smaller than those of the previously known minihalos, which in some cases extend to $\sim 300 \mathrm{kpc}$ from the center. 

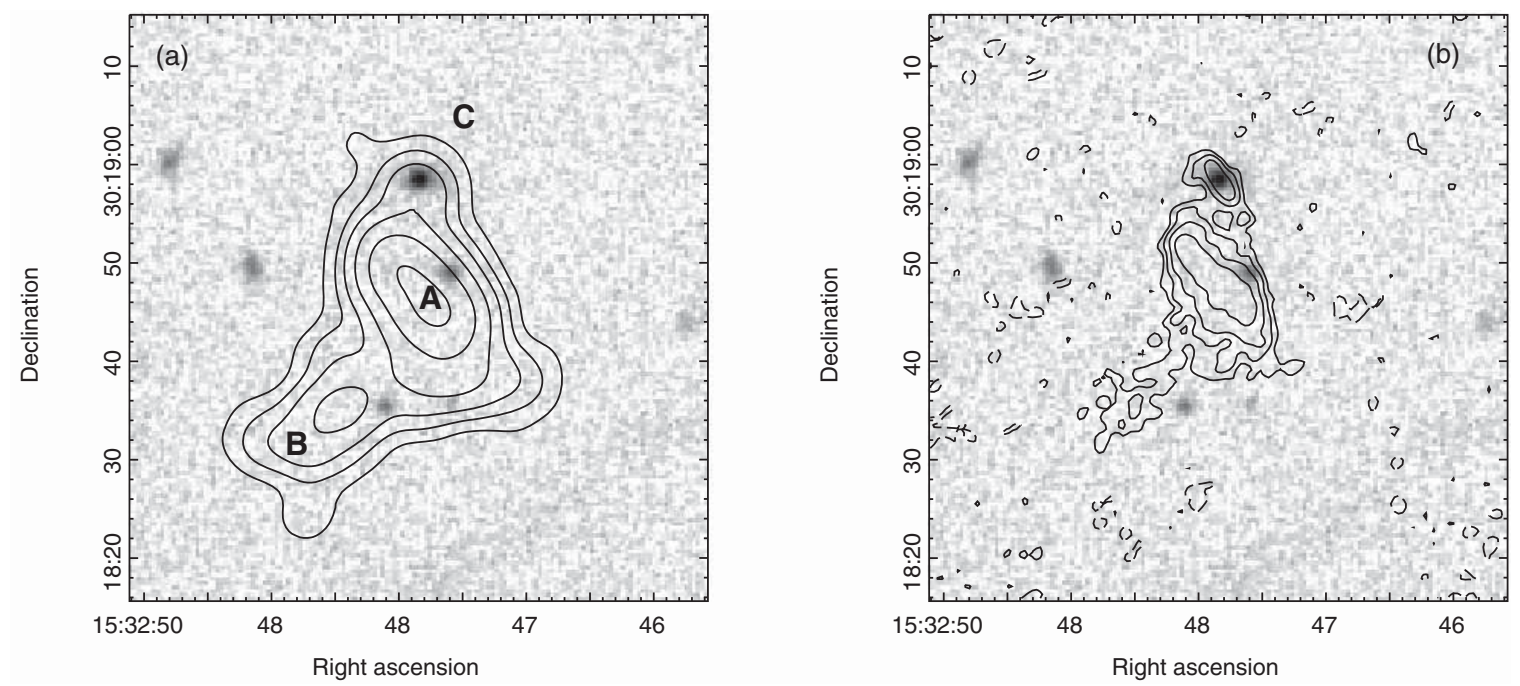

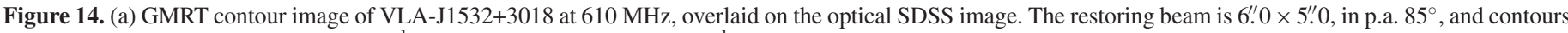

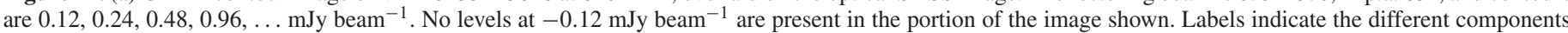

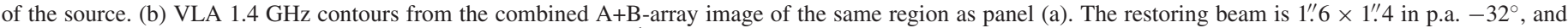
contours are -0.04 (dashed), 0.04, 0.08, 0.16, 0.32, . .mJy beam ${ }^{-1}$.

Combining our new detections with the previously known minihalos results in a total sample of 21 minihalos. We briefly compared radio properties of these clusters with their global $\mathrm{X}$-ray temperatures and total masses estimated from Planck observations. While proper statistical analysis should include nondetections (S. Giacintucci et al., in preparation), we found that clusters hosting minihalos tend to be hot $(T \gtrsim 5 \mathrm{keV})$ and massive systems. Beyond that, we did not see any clear correlation between the minihalo radio luminosity and the cluster global temperature or total mass, in contrast with the behavior of the giant radio halos in merging clusters, whose radio power correlates with the cluster mass (e.g., Cassano et al. 2013). In a subsequent article, we will investigate possible correlations with thermodynamical properties of the cool cores rather than the global cluster properties.

Chandra X-ray images indicate the presence of cold fronts and gas sloshing in the cores of most of our clusters. The minihalos are often (though not always) contained within the regions bounded by the sloshing cold fronts, as previously observed in other minihalo clusters (e.g., Mazzotta \& Giacintucci 2008; ZuHone et al. 2013; S. Giacintucci et al., in preparation). This supports the hypothesis that radio-emitting electrons are reaccelerated by turbulence generated by sloshing (ZuHone et al. 2013). We also argue that simple advection of radio-emitting electrons by the sloshing gas (without the need for reacceleration) may play a role in the formation of the less-extended minihalos.

S.G. acknowledges the support of NASA through Einstein Postdoctoral Fellowship PF0-110071 awarded by the Chandra $\mathrm{X}$-ray Center (CXC), which is operated by SAO. Basic research in radio astronomy at the Naval Research Laboratory is supported by 6.1 base funding. The National Radio Astronomy Observatory is a facility of the National Science Foundation operated under cooperative agreement by Associated Universities, Inc. This research work has used TIFR GMRT Sky Survey (http://tgss.ncra.tifr.res.in) data products. The authors thank the anonymous referee, whose comments and suggestions improved the paper.

\section{APPENDIX}

\section{THE RADIO SOURCE VLA-J1532+3018 IN THE FIELD OF RXJ 1532.9+3021}

An extended radio source with complex morphology is located $\sim 2$ '.6 southwest of the center of the minihalo cluster RXJ 1532.9+3021 (Figure 13). Hereinafter, we refer to this source as VLA-J1532+3018. The GMRT image at $610 \mathrm{MHz}$ (Venturi et al. 2008) and VLA 1.4 GHz image from the combined $\mathrm{A}+\mathrm{B}$ array (Table 2) are shown in Figure 14. Both images are overlaid as contours on the optical red band image from the SDSS. At $610 \mathrm{MHz}$ (panel (a)), three main components can be seen: a bright central component (labeled A) that is mostly elongated in the NE-SW axis; an extended spur-like feature toward SE (B); and a peak of emission to the north (C), coincident with a bright optical galaxy.

At higher resolution (panel (b)), $\mathrm{C}$ is resolved into an extended source with a southwest to northeast major axis. The source is clearly associated with the galaxy SDSS J153247.33+301858 with $z_{\text {phot }}=0.35 \pm 0.03$. The central component A appears as a double-lobe radio galaxy, although no clear jets or a compact central core are visible. The emission in B has very low surface brightness and is apparently connected to A in the form of a tail. The optical galaxy SDSS J153247.09+301849 ( $z_{\text {phot }}=0.36 \pm 0.03$ ) is located within the radio contours on the western side of $\mathrm{A}$, but its association with the radio source is uncertain. $\mathrm{A}$ and $\mathrm{C}$ are also detected, with similar morphology, in the images at $4.9 \mathrm{GHz}$ and $8.5 \mathrm{GHz}$ (not shown here; see Table 2 for details), while for region $\mathrm{B}$ only the emission coincident with the $610 \mathrm{MHz}$ peak is visible at $4.9 \mathrm{GHz}$, and no emission is detected at $8.5 \mathrm{GHz}$.

We interpret VLA-J1532+3018 as the blend of the radio galaxy B and a possible second radio galaxy (A), located at approximately the same redshift. This latter may be a tailed radio source with both jets bent to form a tail toward the SE (C). Assuming $z=0.36$, the length of the tail is $\sim 100 \mathrm{kpc}$. A summary of the properties of VLA-J1532+3018 is provided in Table 6.

Tailed radio galaxies are typically members of groups or clusters of galaxies. Therefore, we searched the literature 
Table 6

Properties of VLA-J1532+3018

\begin{tabular}{|c|c|c|c|c|c|c|c|c|c|}
\hline Component & $\begin{array}{l}\text { Optical id. } \\
\text { (SDSS) }\end{array}$ & $z_{\text {phot }}$ & $\begin{array}{l}S_{610 \mathrm{MHz}} \\
(\mathrm{mJy})\end{array}$ & $\begin{array}{l}S_{1.4 \mathrm{GHz}} \\
(\mathrm{mJy})\end{array}$ & $\begin{array}{l}S_{4.9 \mathrm{GHz}} \\
(\mathrm{mJy})\end{array}$ & $\begin{array}{l}S_{8.5 \mathrm{GHz}} \\
(\mathrm{mJy})\end{array}$ & $\alpha$ & $\begin{array}{c}P_{1.4 \mathrm{GHz}} \\
\left(10^{24} \mathrm{~W} \mathrm{~Hz}^{-1}\right)\end{array}$ & $\begin{array}{c}\text { Size } \\
(\mathrm{kpc} \times \mathrm{kpc})\end{array}$ \\
\hline$A+B$ & $\mathrm{~J} 153247.09+301849^{\mathrm{a}}$ & $0.36 \pm 0.03$ & $25.0 \pm 1.3$ & $13.6 \pm 0.7$ & $4.7 \pm 0.2$ & $2.1 \pm 0.1$ & $0.94 \pm 0.03$ & $6.0 \pm 0.3^{\mathrm{b}}$ & $70 \times 240^{b}$ \\
\hline $\mathrm{C}$ & $\mathrm{J} 153247.33+301858$ & $0.35 \pm 0.03$ & $2.0 \pm 0.1$ & $1.50 \pm 0.08$ & $1.00 \pm 0.05$ & $0.71 \pm 0.04$ & $0.39 \pm 0.03$ & $0.62 \pm 0.03$ & $15 \times 30$ \\
\hline
\end{tabular}

Notes.

${ }^{a}$ Uncertain identification.

${ }^{\mathrm{b}}$ Assuming $z_{\text {phot }}=0.36$.

for indications of a group/cluster at the position of VLA$\mathrm{J} 1532+3018$ and found an optically selected galaxy cluster GMBCG J233.19725+30.31626 at $z_{\text {phot }}=0.358 \pm 0.034$, with $\sim 10$ possible member galaxies (Hao et al. 2010) and a lowsignificance X-ray enhancement in the Chandra image. The cluster is at similar distance as RX J1532.9+3021 $(z=0.36$; Table 1), and their separation in the plane of the sky is approximately $800 \mathrm{kpc}$.

\section{REFERENCES}

Ascasibar, Y., \& Markevitch, M. 2006, ApJ, 650, 102

Bagchi, J., Jacob, J., Gopal-Krishna,, et al. 2009, MNRAS, 399, 601

Becker, R. H., White, R. L., \& Helfand, D. J. 1995, ApJ, 450, 559

Bourdin, H., \& Mazzotta, P. 2008, A\&A, 479, 307

Burns, J. O., Sulkanen, M. E., Gisler, G. R., \& Perley, R. A. 1992, ApJL, 388, L49

Carlstrom, J. E., Holder, G. P., \& Reese, E. D. 2002, ARA\&A, 40, 643

Cassano, R., Brunetti, G., Setti, G., Govoni, F., \& Dolag, K. 2007, MNRAS, 378,1565

Cassano, R., Ettori, S., Brunetti, G., et al. 2013, ApJ, 777, 141

Cassano, R., Gitti, M., \& Brunetti, G. 2008, A\&A, 486, L31

Cavagnolo, K. W., Donahue, M., Voit, G. M., \& Sun, M. 2008, ApJ, 682, 821

Cavagnolo, K. W., Donahue, M., Voit, G. M., \& Sun, M. 2009, ApJS, 182, 12

Chen, Y., Reiprich, T. H., Böhringer, H., Ik ebe, Y., \& Zhang, Y.-Y. 2007, A\&A, 466, 805

Clarke, T. E. 2004, JKAS, 37, 337

Clarke, T. E., Blanton, E. L., \& Sarazin, C. L. 2004, ApJ, 616, 178

Clarke, T. E., Blanton, E. L., Sarazin, C. L., et al. 2009, ApJ, 697, 1481

Condon, J. J., Cotton, W. D., Greisen, E. W., et al. 1998, AJ, 115, 1693

Crawford, C. S., Allen, S. W., Ebeling, H., Edge, A. C., \& Fabian, A. C. 1999, MNRAS, 306, 857

David, L. P., Jones, C., Forman, W., et al. 2009, ApJ, 705, 624

Ebeling, H., Edge, A. C., Bohringer, H., et al. 1998, MNRAS, 301, 881

Ehlert, S., Allen, S. W., von der Linden, A., et al. 2011, MNRAS, 411, 1641

Ettori, S., Fabian, A. C., Allen, S. W., \& Johnstone, R. M. 2002, MNRAS, 331,635

Ettori, S., Gastaldello, F., Gitti, M., et al. 2013, A\&A, 555, A93

Fabian, A. C., Sanders, J. S., Allen, S. W., et al. 2011, MNRAS, 418, 2154

Farnsworth, D., Rudnick, L., Brown, S., \& Brunetti, G. 2013, ApJ, 779, 189

Feretti, L., Giovannini, G., Govoni, F., \& Murgia, M. 2012, A\&A Rev., 20, 54

Forman, W., Jones, C., Markevitch, M., Vikhlinin, A., \& Churazov, E. 2002, arXiv:astro-ph/0207165

Fujita, Y., Kohri, K., Yamazaki, R., \& Kino, M. 2007, ApJL, 663, L61

Fujita, Y., Matsumoto, T., \& Wada, K. 2004, ApJL, 612, L9

Fujita, Y., \& Ohira, Y. 2013, MNRAS, 428, 599

Gastaldello, F., Di Gesu, L., Ghizzardi, S., et al. 2013, ApJ, 770, 56

Ge, J. P., \& Owen, F. N. 1993, AJ, 105, 778

Ghizzardi, S., Rossetti, M., \& Molendi, S. 2010, A\&A, 516, A32

Giacintucci, S., Markevitch, M., Brunetti, G., Cassano, R., \& Venturi, T. 2011a, A\&A, 525, L10

Giacintucci, S., O’Sullivan, E., Clarke, T. E., et al. 2012, ApJ, 755, 172

Giacintucci, S., O'Sullivan, E., Vrtilek, J., et al. 2011b, ApJ, 732, 95

Giovannini, G., \& Feretti, L. 2000, NewA, 5, 335
Gitti, M. 2013, MNRAS, 436, L84

Gitti, M., Brunetti, G., Feretti, L., \& Setti, G. 2004, A\&A, 417, 1

Gitti, M., Brunetti, G., \& Setti, G. 2002, A\&A, 386, 456

Govoni, F., Murgia, M., Markevitch, M., et al. 2009, A\&A, 499, 371

Gregory, P. C., \& Condon, J. J. 1991, ApJS, 75, 1011

Hao, J., McKay, T. A., Koester, B. P., et al. 2010, ApJS, 191, 254

Hlavacek-Larrondo, J., Allen, S. W., Taylor, G. B., et al. 2013, ApJ, 777, 163

Hudson, D. S., Mittal, R., Reiprich, T. H., et al. 2010, A\&A, 513, A37

Ikebe, Y., Reiprich, T. H., Böhringer, H., Tanaka, Y., \& Kitayama, T. 2002, A\&A, 383,773

Jaffe, W. J. 1977, ApJ, 212, 1

Kale, R., Venturi, T., Giacintucci, S., et al. 2013, A\&A, 557, A99

Kausch, W., Gitti, M., Erben, T., \& Schindler, S. 2007, A\&A, 471, 31

Keshet, U. 2010, arXiv:1011.0729

Keshet, U., \& Loeb, A. 2010, ApJ, 722, 737

Laganá, T. F., Andrade-Santos, F., \& Lima Neto, G. B. 2010, A\&A, 511, A15

Lane, W. M., Cotton, W. D., Helmboldt, J. F., \& Kassim, N. E. 2012, RaSc, 47, 0

Markevitch, M., \& Vikhlinin, A. 2007, PhR, 443, 1

Markevitch, M., Vikhlinin, A., \& Forman, W. R. 2003, ASPC, 301, 37

Markevitch, M., Vikhlinin, A., \& Mazzotta, P. 2001, ApJL, 562, L153

Maughan, B. J., Jones, C., Forman, W., \& Van Speybroeck, L. 2008, ApJS, 174,117

Mazzotta, P., Edge, A. C., \& Markevitch, M. 2003, ApJ, 596, 190

Mazzotta, P., \& Giacintucci, S. 2008, ApJL, 675, L9

Mazzotta, P., Markevitch, M., Vikhlinin, A., et al. 2001, ApJ, 555, 205

Murgia, M., Eckert, D., Govoni, F., et al. 2010, A\&A, 514, A76

Murgia, M., Govoni, F., Markevitch, M., et al. 2009, A\&A, 499, 679

Murgia, M., Parma, P., Mack, K.-H., et al. 2011, A\&A, 526, A148

Owers, M. S., Nulsen, P. E. J., Couch, W. J., \& Markevitch, M. 2009, ApJ, 704, 1349

Paterno-Mahler, R., Blanton, E. L., Randall, S. W., \& Clarke, T. E. 2013, ApJ, 773,114

Pedlar, A., Ghataure, H. S., Davies, R. D., et al. 1990, MNRAS, 246, 477

Perley, R. A., \& Butler, B. J. 2013, ApJS, 204, 19

Pfrommer, C., \& Enßlin, T. A. 2004, A\&A, 413, 17

Planck Collaboration, Ade, P. A. R., Aghanim, N., et al. 2013, arXiv:1303.5089

Randall, S. W., Forman, W. R., Giacintucci, S., et al. 2011, ApJ, 726, 86

Rengelink, R. B., Tang, Y., de Bruyn, A. G., et al. 1997, A\&AS, 124, 259

Rossetti, M., Eckert, D., De Grandi, S., et al. 2013, A\&A, 556, A44

Saikia, D. J., \& Jamrozy, M. 2009, BASI, 37, 63

Sanders, J. S., Fabian, A. C., \& Taylor, G. B. 2005, MNRAS, 356, 1022

Sanders, J. S., Fabian, A. C., \& Taylor, G. B. 2009, MNRAS, 393, 71

Sarazin, C. L., Baum, S. A., \& O’Dea, C. P. 1995, ApJ, 451, 125

Scaife, A. M. M., \& Heald, G. H. 2012, MNRAS, 423, L30

Sijbring, L. G. 1993, PhD thesis, University of Groningen

Sun, M., Jones, C., Murray, S. S., et al. 2003, ApJ, 587, 619

Taylor, G. B., Fabian, A. C., \& Allen, S. W. 2002, MNRAS, 334, 769

Vazza, F., Roediger, E., \& Brüggen, M. 2012, A\&A, 544, A103

Venturi, T., Giacintucci, S., Brunetti, G., et al. 2007, A\&A, 463, 937

Venturi, T., Giacintucci, S., Dallacasa, D., et al. 2008, A\&A, 484, 327

Venturi, T., Rossetti, M., Bardelli, S., et al. 2013, A\&A, 558, A146

Zabludoff, A. I., Huchra, J. P., \& Geller, M. J. 1990, ApJS, 74, 1

ZuHone, J. A., Markevitch, M., Brunetti, G., \& Giacintucci, S. 2013, ApJ, 762,78

ZuHone, J. A., Markevitch, M., \& Johnson, R. E. 2010, ApJ, 717, 908 